\title{
PARALLELISM BETWEEN CHARGE REDISTRIBUTION AND DELOCALIZATION. APPLICATIONS TO ORGANIC REACTIONS
}

\author{
V. Gineitytè \\ Institute of Theoretical Physics and Astronomy, Vilnius University, A. Goštauto 12, LT-01108 Vilnius, Lithuania \\ E-mail: viktorija.gineityte@tfai.vu.lt
}

Received 1 April 2011; revised 15 June 2011; accepted 21 June 2011

\begin{abstract}
The study is devoted to interrelations between two perturbative expansions for molecules and molecular systems, namely between terms of power series for one-electron density matrices on the one hand, and those for respective representation matrices of non-canonical (localized) molecular orbitals (MOs) on the other hand, as well as to the relevant implications. As the most outstanding example of the latter, simple proportionalities are established between alterations in populations of basis orbitals due to chemical interaction and delocalization coefficients of respective localized MOs (LMOs). The proof of these proportionalities is valid for members of power series to within the fifth order inclusive that were shown previously to be sufficient for investigations of the most important organic reactions. As a result, classical interpretations of early stages of these reactions in terms of shifts of respective localized pairs of electrons (cf. the so-called 'curly arrow chemistry') acquire a quantum-chemical support. Moreover, the results of the present study allow comparisons of relative extents of delocalization of LMOs for alternative routes of the same process. On this basis, predominant (allowed) routes of organic reactions are shown to be characterized by enhanced delocalization of respective principal pairs of electrons as compared to alternative (forbidden) routes.
\end{abstract}

Keywords: localized molecular orbitals, Brillouin theorem, organic reactions, delocalization, curly arrow chemistry

PACS: $31.10 .+\mathrm{z}, 31.15 . \mathrm{Md}$

\section{Introduction}

The local perspective on chemical reactivity is known to be prevalent in the classical chemistry [1-6]. In particular, a definite functional group is usually regarded as taking part in the given process directly and it is referred to as the reaction centre [1]. Again, the remaining parts of molecules are supposed to participate in the same process indirectly by exerting certain electron-donating or accepting effects upon this centre. Extinction of these effects when the distance between the given fragment and the respective reaction centre becomes larger is also among the expectations.

To enable the quantum-chemical study of the abovespecified different roles, the so-called semilocalized approach to chemical reactivity has been suggested and applied [7-9]. The approach is based on expressions for one-electron density matrices (DMs) of molecules and molecular systems derived previously in the form of power series [10]. Electron density redistributions between orbitals of elementary fragments of the whole reacting system (the so-called fragmental orbitals, FOs) was the principal characteristic under study in this approach. (More efficient charge redistributions were shown to imply a more significant stabilization of the whole system [11].) Individual chemical bonds along with lone electron pairs (if any) usually play the role of fragments, although other types of the latter (e.g. phenyl rings) are also possible. Anyway, FOs always may be classified into initially-doubly-occupied (bonding) and initially-vacant (antibonding) orbitals. Applications of the semilocalized approach to the case of two weakly-interacting molecules of any structure [8] show that local charge redistributions inside and between the relevant reaction centres are expressible in terms of power series of lower orders, whilst redistributions between orbitals of more remote fragments are represented by terms of higher orders. Thus, the decisive role of redistributions of the former type has been supported. Moreover, the predominant routes of specific reactions have been shown to be accompanied by more efficient charge redistributions between the relevant principal FOs [9].

Interpretations of organic reactions in terms of shifts of separate pairs of electrons [1, 4-6, 12] (cf. the so-called 'curly arrow chemistry') also represent the above-described local perspective. Models of this type are based on the fundamental classical concept of elec- 
tron pairs pertinent to individual chemical bonds [4, 5, 13], which is commonly ascribed to Lewis [14]. It is also essential in the present context that only a few pairs (usually those referring to the reaction centre) are assumed to undergo essential shifts and thereby to play an important role in a given reaction, whilst the remaining electrons are considered inactive. The original form of the above-discussed semilocalized approach [7, 8], however, is still insufficient to reflect the concepts underlying the 'curly arrow chemistry' and/or to find their quantum- chemical analogues. This important point deserves a more detailed discussion.

The occupation number (population) of a certain basis function is defined in quantum chemistry as a sum of increments of all occupied one-electron states (molecular orbitals) and thereby of all electrons of the given system (see e.g. [15, 16]). This general definition evidently embraces populations of FOs of the semilocalized approach too. To achieve the above-specified ends, however, a relation is required between an alteration in the population of a certain basis orbital due to chemical interaction and reshaping of the respective single pair of electrons. Such a relation (if established) would provide us with a simple interpretation of the abovediscussed local charge redistributions in terms of shifts of respective local pairs of electrons.

In the present study, we will confine ourselves to the most common case of molecules representable by a single principal Lewis structure (electron dot diagram) [13, 17, 18]. A shift and/or reshaping of a certain pair of electrons may be then ascribed to the changing extent of its delocalization. Moreover, a kind of parallelism between charge redistribution and delocalization seems to be among immanent features of the above-specified systems.

To illustrate these statements, let us assume for a moment that contributions of extra Lewis structures may be ignored in the initial compounds. The relevant initial charge distribution is then defined by superposition of increments of localized pairs of electrons attached to separate bonds and/or other fragments, whereas the subsequent charge redistribution due to chemical interaction necessarily implies a certain extent of delocalization of these pairs, especially of those participating in the given process directly. (Note that a shift of electrons along the axis of the 'own' bond, cf. the so-called bond polarization, is equivalent to an intrabond delocalization of the relevant bonding orbital over the antibonding one [19].) It is also worth adding here that parallelism between charge redistribution and delocalization is assumed implicitly when discussing relative stabili- ties of particular reaction intermediates (especially of ions) [2, 20]. Indeed, stability of such an intermediate is usually traced back to better conditions for delocalization and/or for redistribution of an initially-localized positive or negative charge. Besides, both charge redistribution and delocalization are supposed to be weak effects in these discussions [2].

In summary, our principal task consists in relating the actual population of a certain initially-doublyoccupied FO to the extent of delocalization of the respective single pair of electrons.

Quantum chemical descriptions of many-electron systems in terms of predominantly localized (or weaklydelocalized) pairs of electrons are known to be achievable in the framework of the non-canonical method of molecular orbitals (MOs) [21], where various types of the so-called localized MOs (LMOs) may be constructed (see e.g. [22-25] for review). (Note that the most popular canonical MOs (CMOs) usually are delocalized over the whole system for symmetry reasons $[16,21]$.) Moreover, LMOs may be obtained both directly [10, 19, 24-42] and indirectly (i. e. by transforming the set of CMOs into that of LMOs using various localization criteria [43-46]). Invoking the Brillouin theorem $[10,19,26-29,40-42]$ and related approaches $[24,35,38,39]$ is among the most efficient ways of their direct derivation.

The power series for the one-electron DM [10] underlying the semilocalized approach of Refs. [7, 8] resulted from a direct solution of the so-called commutation equation for this matrix [47]. Analysis of the latter in comparison to the Brillouin theorem has been carried out in Ref. [10] to within the second order members of perturbative expansions inclusive. It turns out that these two fundamental non-canonical one-electron problems yield similar intermediate equations. Consequently, the first three terms of power series for the relevant representation matrices (i.e. for the chargebond order (CBO) matrix $\mathbf{P}$ and for the respective LMO representation matrix $\mathbf{C}$ ) have been shown to consist of the same submatrices (blocks) and thereby to be interrelated. Proportionality between the actual population of a certain basis function (FO) and the so-called delocalization coefficient [42] of the respective single LMO then straightforwardly followed from the abovementioned interrelation and also accordingly embraced terms referring to the order parameter $k=0,1$, and 2 .

It should be mentioned, however, that members of the power series for the $\mathrm{CBO}$ matrix $\mathbf{P}$ just of higher orders have been shown to play the decisive role when describing chemical reactions (terms to within the fifth 
order inclusive prove to be important as discussed in Ref. [9] and in Sec. 5). Meanwhile, the validity of the above-overviewed results for $k>2$ is far from being self-evident. Thus, the aim of the present study is to extend both interrelations between LMOs and DM and their implications to the case of higher values of the order parameter (i.e. for $k=3,4$, and 5). On this basis, we are about to demonstrate a possibility of interpretation of organic reactions in terms of variable extents of delocalization of LMOs.

In spite of the above-formulated seemingly modest aim, the present study is not a direct continuation of the previous analysis [10]. Quite the reverse, the overall scheme of the paper is chosen so as to emphasize new important aspects of the whole problem. Early achievements are also considered from another perspective. To outline the latter, let us start with the notation that a set of non-canonical (localized) MOs is not a unique characteristic of a molecule [16, 21, 25], in contrast to both CMOs and DM. It is no surprise, therefore, that the Brillouin theorem as such does not yield a unique set of LMOs, and some additional conditions and/or criteria are required to obtain them. In this connection, the original study [10] started with solution of the commutation equation for the CBO matrix $\mathbf{P}$, whilst the LMO representation matrix $\mathbf{C}$ was chosen so as to resemble the matrix $\mathbf{P}$ as closely as possible in the subsequent application of the Brillouin theorem. That is why the zero order member $\left(\mathbf{C}_{(0)}\right)$ of the power series for the matrix $\mathbf{C}$ was taken equal to the unit matrix (I) and thereby LMOs of the basis-orbital-and-tail constitution were actually chosen. Moreover, submatrices (blocks) occupying the diagonal positions within corrections $\mathbf{C}_{(1)}$ and $\mathbf{C}_{(2)}$ were assumed to be Hermitian (symmetric) matrices. This scheme of analysis may create an impression that derivation of the LMO representation matrix is a kind of attachment to the principal problem of obtaining the unique DM.

The overall situation, however, changed after revealing the nature of perturbative expansions of Refs. [10, 19, 41, 42]. Indeed, the latter were shown [48, 49] to be based on a generalization of the standard RayleighSchrödinger perturbation theory (RSPT) [16, 50], wherein the usual Hamiltonian matrix elements are replaced by multidimensional parameters coinciding with submatrices (blocks) of the initial Hamiltonian matrix. In this new context it appeared [48] that the abovementioned two additional conditions (i. e. $\mathbf{C}_{(0)}=\mathbf{I}$ and the Hermitian nature of particular blocks of corrections $\mathbf{C}_{(1)}$ and $\mathbf{C}_{(2)}$ ) have nothing to do either with the Brillouin theorem itself or with similarity between matri- ces $\mathbf{C}$ and $\mathbf{P}$ : these prove to be analogous generalizations of the relevant conditions of the usual RSPT [50]. This implies that derivation of the LMO representation matrix may be carried out without any reference to the DM. Moreover, the DM and/or the CBO matrix $\mathbf{P}$ was subsequently shown to be obtainable on the basis of a projector to the set of occupied LMOs [51], i. e. as a second step of the whole theory as usual. In summary, the two principal non-canonical one-electron problems become mutually independent and equivalent.

As already mentioned, the present study is orientated mostly towards applications of LMOs to chemical reactions. In this connection, we start with the direct obtaining of LMOs on the basis of the Brillouin theorem (Sec. 2) and derive formulae for members of the power series for the matrix $\mathbf{C}$ to within the fifth order $(k=5)$ inclusive. Respective members of perturbative expansions for delocalization coefficients of LMOs are also exhibited in the same section. Thereupon, we turn to the general definition of an occupation number (population) of a certain basis function as a sum of increments of all occupied LMOs and obtain an expression for this characteristic via the delocalization coefficient of the respective single LMO (Sec. 3). Demonstration of how the increments of the remaining LMOs vanish in this expression is also among aims of this derivation. Section 4 contains a discussion of the practically important case of the theory corresponding to all interorbital interactions of the first order magnitude. The final section is devoted to consideration of specific organic reactions.

\section{Expressions for localized molecular orbitals and their principal properties}

Let the total basis set of FOs $\{\Phi\}$ to consist of two subsets $\left\{\Phi_{1}\right\}$ and $\left\{\Phi_{2}\right\}$, the first one containing initially-occupied FOs (IOFOs) and the second subset embracing the initially-vacant orbitals (IVFOs). Convenient subscripts $(+)$ and $(-)$ introduced and used previously [7-11, 19, 41, 42, 48, 49, 51] will also be preserved for individual orbitals so that $\varphi_{(+) i}$ and $\varphi_{(-) l}$ will stand for the $i$ th IOFO and for the $l$ th IVFO, respectively. Orbitals of different subsets are assumed to be separated by a substantial energy gap in addition and the intersubset interactions are regarded as first order terms versus this gap. The actual number of molecules is not essential here. 
Let the total one-electron Hamiltonian matrix of our system (H) be expressed as a sum of zero- and firstorder matrices, i. e.,

$$
\mathbf{H}=\mathbf{H}_{(0)}+\mathbf{H}_{(1)},
$$

where

$$
\mathbf{H}_{(0)}=\left|\begin{array}{cc}
\mathbf{E}_{(+)} & \mathbf{0} \\
\mathbf{0} & -\mathbf{E}_{(-)}
\end{array}\right|, \quad \mathbf{H}_{(1)}=\left|\begin{array}{cc}
\mathbf{S} & \mathbf{R} \\
\mathbf{R}^{+} & \mathbf{Q}
\end{array}\right| .
$$

Submatrices $\mathbf{E}_{(+)}+\mathbf{S}$ and $-\mathbf{E}_{(-)}+\mathbf{Q}$ of the matrix $\mathbf{H}$ contain intrasubset interactions (resonance parameters) along with one-electron energies of FOs, whilst the off-diagonal block $\mathbf{R}$ involves intersubset interactions. The minus sign in front of $\mathbf{E}_{(-)}$is introduced for convenience. The superscript + here and below designates the Hermitian-conjugate matrix. Besides, zero order intrasubset interactions are generally allowed in Eq. (2) and these are included into submatrices $\mathbf{E}_{(+)}$ and $\mathbf{E}_{(-)}$.

Let us introduce now an additional unitarity condition for the LMO representation $\mathbf{C}$ as previously [10, 19, 41, 42, 48, 49, 51], i. e.,

$$
\mathbf{C}^{+} \mathbf{C}=\mathbf{I},
$$

and turn to the Brillouin theorem [21, 25]. Among particular forms of this theorem there is a zero value requirement for an off-diagonal element of the Fockian (or Hamiltonian) operator referring to an occupied and a vacant one-electron orbital (MO). In its matrix form, this requirement resolves itself into the zero matrix condition for the occupied-vacant (i. e. off-diagonal) block (submatrix) of the total Fockian (Hamiltonian) matrix in the basis of LMOs being sought [10, 41, 42]. For the matrix $\mathbf{H}$ of Eqs. (1) and (2), we then obtain the following requirement:

$$
\left\{\mathbf{C}^{+} \mathbf{H C}\right\}_{12}=\left\{\mathbf{C}^{+} \mathbf{H C}\right\}_{21}=\mathbf{0},
$$

where Eq. (3) is also taken into consideration. The subscripts 12 and 21 stand here for the off-diagonal block of the total matrix product within the braces. An alternative form of Eq. (4) coincides with the blockdiagonality condition for the transformed Hamiltonian matrix, namely,

$$
\mathbf{H}^{\prime}=\mathbf{C}^{+} \mathbf{H C}=\left|\begin{array}{cc}
\mathbf{E}_{1} & \mathbf{0} \\
\mathbf{0} & \mathbf{E}_{2}
\end{array}\right|,
$$

where $\mathbf{E}_{1}$ and $\mathbf{E}_{2}$ are the so-called eigenblocks of the matrix $\mathbf{H}$ [42]. The relation (5) represents the blockdiagonalization problem for our Hamiltonian matrix.
It is evident that the total set of LMOs $\{\Psi\}$ is also divisible into two subsets $\left\{\Psi_{1}\right\}$ and $\left\{\Psi_{2}\right\}$ containing occupied and vacant orbitals, respectively. Further, both LMOs and FOs may be collected into row-matrices, the latter being designated by respective ket-vectors, e. g. $\left|\Psi_{1}\right\rangle,\left|\Psi_{2}\right\rangle,\left|\Phi_{1}\right\rangle$, etc. Bra-vectors (e.g. $\left.\left\langle\Psi_{1}\right|\right)$ will accordingly stand for column-matrices of the relevant orbitals. The interrelation between the total ket-vector of LMOs $(|\Psi\rangle)$ and that of FOs $(|\Phi\rangle)$ is then as follows:

$$
|\Psi\rangle=|\Phi\rangle \mathbf{C} .
$$

Let the total matrix $\mathbf{C}$ be represented in terms of four submatrices (blocks), too,

$$
\mathbf{C}=\left|\begin{array}{ll}
\mathbf{C}_{11} & \mathbf{C}_{12} \\
\mathbf{C}_{21} & \mathbf{C}_{22}
\end{array}\right|
$$

Substituting Eq. (7) into Eq. (6) then yields the following expression for the ket-vector of occupied LMOs:

$$
\left|\Psi_{1}\right\rangle=\left|\Phi_{1}\right\rangle \mathbf{C}_{11}+\left|\Phi_{2}\right\rangle \mathbf{C}_{21} .
$$

The vector of vacant LMOs $\left|\Psi_{2}\right\rangle$ may also be expressed analogously.

Constitution of the initial Hamiltonian matrix $\mathbf{H}$ shown in Eq. (1) allows the LMO representation matrix $\mathbf{C}$ to be sought in the form of power series, i. e. as a sum of corrections $\mathbf{C}_{(k)}$ of various orders $(k)$. The same form also refers to separate submatrices of the matrix C. As already mentioned, we will confine ourselves to LMOs of the basis-orbital-and-tail constitution. Consequently, the equality $\mathbf{C}_{(0)}=\mathbf{I}$ will be accepted. In summary, we will look for the matrix $\mathbf{C}$ of the following form:

$$
\mathbf{C}=\mathbf{I}+\sum_{k=1}^{\infty} \mathbf{C}_{(k)} .
$$

For separate blocks of the LMO representation matrix C, we accordingly obtain

$$
\begin{aligned}
& \mathbf{C}_{11}=\mathbf{I}+\sum_{k=1}^{\infty} \mathbf{C}_{11}^{(k)}, \quad \mathbf{C}_{22}=\mathbf{I}+\sum_{k=1}^{\infty} \mathbf{C}_{22}^{(k)}, \\
& \mathbf{C}_{12}=\sum_{k=1}^{\infty} \mathbf{C}_{12}^{(k)}, \quad \mathbf{C}_{21}=\sum_{k=1}^{\infty} \mathbf{C}_{21}^{(k)},
\end{aligned}
$$

where the order parameter $(k)$ now takes the upper position for convenience.

One-to-one correspondence between FOs and LMOs may be easily seen after substituting Eq. (10) into Eqs. (7) and (8). This allows the same subscripts (i.e. $(+) i,(+) j,(-) l$, etc) to be used to designate both types 
of orbitals. As for instance, an individual occupied LMO $\psi_{(+) i}$ takes the form

$$
\begin{aligned}
& \psi_{(+) i}=\varphi_{(+) i} \\
& +\sum_{k=1}^{\infty}\left[\sum_{(+) j}^{(\text {IOFOs })} \varphi_{(+) j} C_{11, j i}^{(k)}+\sum_{(-) l}^{(\text {IVFOs })} \varphi_{(-) l} C_{21, l i}^{(k)}\right]
\end{aligned}
$$

and contains the principal (i.e. zero order) contribution of the respective $\mathrm{FO} \varphi_{(+) i}$ and a tail consisting of increments of other FOs. Sums over $(+) j$ and $(-) l$ embrace here the initially-occupied and initially-vacant FOs, respectively, as indicated above the sum signs, whilst $C_{11, j i}^{(k)}$ and $C_{21, l i}^{(k)}$ correspondingly stand for elements of submatrices $\mathbf{C}_{11}^{(k)}$ and $\mathbf{C}_{21}^{(k)}$ of Eq. (10). On the basis of the above formulae we may also assert that elements of submatrices $\mathbf{C}_{21}^{(k)}$ and $\mathbf{C}_{12}^{(k)}$ reflect tails of LMOs of the intersubset type (i. e. the intersubset delocalization), whereas those of the remaining submatrices $\left(\mathbf{C}_{11}^{(k)}\right.$ and $\left.\mathbf{C}_{22}^{(k)}\right)$ represent tails of the intrasubset type (intrasubset delocalization). Inasmuch as diagonal elements $C_{11, i i}^{(k)}, k=1,2, \ldots$ describe renormalization of the IOFO $\varphi_{(+) i}$ when building up the respective LMO $\psi_{(+) i}$, the total matrix $\mathbf{C}_{11}$ will be called the renormalization matrix for convenience. The same refers also to the matrix $\mathbf{C}_{22}$.

To derive formulae for individual corrections of Eq. (10), Eqs. (7), (9), and (10) should be substituted into Eqs. (3) and (4). Thereupon, terms of the same order should be collected and studied separately. This procedure is described in the Appendix A in detail. Thus, let us confine ourselves here to the relevant principal results.

The off-diagonal blocks $\mathbf{C}_{12}^{(k)}$ and $\mathbf{C}_{21}^{(k)}$ of corrections $\mathbf{C}_{(k)}(k=1,2,3, \ldots)$ are determined by the Brillouin theorem (block-diagonalization problem) itself. Moreover, these blocks are interrelated and expressible via a single matrix $\widetilde{\mathbf{G}}_{(k)}$ :

$$
\mathbf{C}_{12}^{(k)}=-\mathbf{C}_{21}^{(k)+}=\widetilde{\mathbf{G}}_{(k)} .
$$

Matrices $\widetilde{\mathbf{G}}_{(k)}, k=1,2,3, \ldots$, in turn, are conditioned by specific matrix equations (see Eqs. (A11) and (A14)) containing entire submatrices of our initial Hamiltonian matrix, i.e. $\mathbf{E}_{(+)}, \mathbf{E}_{(-)}, \mathbf{S}, \mathbf{Q}$, and $\mathbf{R}$. In the particular case of diagonal zero order Hamiltonian matrices (i. e. of diagonal submatrices $\mathbf{E}_{(+)}$and $\mathbf{E}_{(-)}$consisting of one-electron energies of FOs only), separate elements $\widetilde{G}_{(k) i l}$ are expressible algebraically as discussed in Sec. 4. The additional symbol ' $\sim$ ' serves here to dis- tinguish between the principal matrices of the present study (i. e. those determining the off-diagonal blocks of the LMO representation matrix $\mathbf{C})$ and matrices $\mathbf{G}_{(k)}$, $k=1,2,3, \ldots$ arising in the off-diagonal positions of corrections $\mathbf{P}_{(k)}$ of the relevant CBO matrix $\mathbf{P}$ [10]. Actually, however, the distinction concerned manifests itself starting with $k=3$ only, i.e. $\widetilde{\mathbf{G}}_{(1)}=\mathbf{G}_{(1)}$ and $\widetilde{\mathbf{G}}_{(2)}=\mathbf{G}_{(2)}$. (Interrelations between matrices $\widetilde{\mathbf{G}}_{(3)}$ and $\mathbf{G}_{(3)}$, as well as between $\widetilde{\mathbf{G}}_{(4)}$ and $\mathbf{G}_{(4)}$ are studied in the Appendix B and exhibited in Eqs. (B8) and (B9).) From Eqs. (11) and (12) we obtain

$$
\begin{aligned}
& \mathbf{C}_{12}=\mathbf{G}_{(1)}+\mathbf{G}_{(2)}+\widetilde{\mathbf{G}}_{(3)}+\widetilde{\mathbf{G}}_{(4)}+\ldots \\
& \mathbf{C}_{21}=-\left(\mathbf{G}_{(1)}^{+}+\mathbf{G}_{(2)}^{+}+\widetilde{\mathbf{G}}_{(3)}^{+}+\widetilde{\mathbf{G}}_{(4)}^{+}+\ldots\right) .
\end{aligned}
$$

Hence, tails of LMOs of the intersubset type prove to be determined by elements of sums of the right-hand sides of Eq. (13). As for instance, the tail of the LMO $\psi_{(+) i}$ over the IVFO $\varphi_{(-) l}$ is represented by the following element:

$$
C_{21, l i}=-\left(G_{(1) l i}^{+}+G_{(2) l i}^{+}+\widetilde{G}_{(3) l i}^{+}+\widetilde{G}_{(4) l i}^{+}+\ldots\right) .
$$

Interpretations of these elements are discussed in Sec. 4.

As opposed to the above-considered submatrices $\mathbf{C}_{12}$ and $\mathbf{C}_{21}$, members of the power series for renormalization matrices $\mathbf{C}_{11}$ and $\mathbf{C}_{22}$ (i. e. for $\mathbf{C}_{11}^{(k)}$ and $\mathbf{C}_{22}^{(k)}$ ) follow from the unitarity condition of Eq. (3) only (see Appendix A). Moreover, these members are expressible algebraically in terms of products of matrices $\widetilde{\mathbf{G}}_{(k)}$ of lower orders as exhibited by Eqs. (A16) and (A23)(A25).

Let us return again to the intersubset tails of LMOs shown in Eq. (14) and define the so-called partial delocalization coefficients [42] of LMOs $\psi_{(+) i}$ and $\psi_{(-) m}$ over particular FOs, e. g. over $\varphi_{(-) l}$ and $\varphi_{(+) j}$, respectively. Let these coefficients be correspondingly denoted by $d_{(+) i,(-) l}$ and $d_{(-) m,(+) j}$. The defitions concerned are as follows:

$$
\begin{aligned}
& d_{(+) i,(-) l}=\left|C_{21, l i}\right|^{2}= \\
& \left|G_{(1) l i}^{+}+G_{(2) l i}^{+}+\widetilde{G}_{(3) l i}^{+}+\widetilde{G}_{(4) l i}^{+}+\ldots\right|^{2}, \\
& d_{(-) m,(+) j}=\left|C_{12, j m}\right|^{2}= \\
& \left|G_{(1) j m}+G_{(2) j m}+\widetilde{G}_{(3) j m}+\widetilde{G}_{(4) j m}+\ldots\right|^{2}
\end{aligned}
$$


It is easily seen that the partial delocalization coefficients are also expressible in the form of power series, i.e.,

$$
\begin{aligned}
& d_{(+) i,(-) l}=\sum_{k=2}^{\infty} d_{(+) i,(-) l}^{(k)}, \\
& d_{(-) m,(+) j}=\sum_{k=2}^{\infty} d_{(-) m,(+) j}^{(k)} .
\end{aligned}
$$

In contrast to Eq. (11), the series of Eq. (16) start with $k=2$. Moreover, from Eq. (15) we obtain

$$
d_{(+) i,(-) l}^{(k)}=d_{(-) l,(+) i}^{(k)}
$$

for any $k$, where

$$
\begin{aligned}
d_{(+) i,(-) l}^{(2)}= & \left|G_{(1) i l}\right|^{2}, \\
d_{(+) i,(-) l}^{(3)}= & G_{(1) i l} G_{(2) i l}^{*}+G_{(1) i l}^{*} G_{(2) i l}, \\
d_{(+) i,(-) l}^{(4)}= & G_{(1) i l} \widetilde{G}_{(3) i l}^{*}+G_{(1) i l}^{*} \widetilde{G}_{(3) i l}+\left|G_{(2) i l}\right|^{2}, \\
d_{(+) i,(-) l}^{(5)}= & G_{(1) i l} \widetilde{G}_{(4) i l}^{*}+G_{(1) i l}^{*} \widetilde{G}_{(4) i l}+G_{(2) i l} \widetilde{G}_{(3) i l}^{*} \\
& +G_{(2) i l}^{*} \widetilde{G}_{(3) i l},
\end{aligned}
$$

and $*$ designates the complex-conjugate counterpart of the respective matrix element. The second order contributions $d_{(+) i,(-) l}^{(2)}$ to partial delocalization coefficients always are positive quantities as Eq. (18) shows. Meanwhile, the signs of the remaining members of the same series cannot be established a priori.

Let us define now the total (intersubset) delocalization coefficients [42] of LMOs $\psi_{(+) i}$ and $\psi_{(-) m}$ over all IVFOs and all IOFOs, respectively, i. e.,

$$
\begin{aligned}
& D_{(+) i}=\sum_{(-) l}^{(\text {IVFOs })} d_{(+) i,(-) l}, \\
& D_{(-) m}=\sum_{(+) j}^{(\text {IOFOs })} d_{(-) m,(+) j} .
\end{aligned}
$$

From Eqs. (15) and (19) it follows that $D_{(+) i}$ and $D_{(-) m}$ actually coincide with diagonal elements of the following matrices:

$$
\mathbf{D}_{(+)}=\mathbf{C}_{21}^{+} \mathbf{C}_{21}, \quad \mathbf{D}_{(-)}=\mathbf{C}_{12}^{+} \mathbf{C}_{12},
$$

i.e. $D_{(+) i} \equiv D_{(+) i i}$ and $D_{(-) m} \equiv D_{(-) m m}$. In this connection, let $\mathbf{D}_{(+)}$and $\mathbf{D}_{(-)}$be further referred to as the intersubset delocalization matrices. Substituting Eq. (13) into Eq. (20) yields members of power series for these matrices. For instance, we obtain

$$
\begin{aligned}
\mathbf{D}_{(+)}^{(2)}= & \mathbf{G}_{(1)} \mathbf{G}_{(1)}^{+}, \\
\mathbf{D}_{(+)}^{(3)}= & \mathbf{G}_{(1)} \mathbf{G}_{(2)}^{+}+\mathbf{G}_{(2)} \mathbf{G}_{(1)}^{+}, \\
\mathbf{D}_{(+)}^{(4)}= & \mathbf{G}_{(1)} \widetilde{\mathbf{G}}_{(3)}^{+}+\widetilde{\mathbf{G}}_{(3)} \mathbf{G}_{(1)}^{+}+\mathbf{G}_{(2)} \mathbf{G}_{(2)}^{+}, \\
\mathbf{D}_{(+)}^{(5)}= & \mathbf{G}_{(1)} \widetilde{\mathbf{G}}_{(4)}^{+}+\widetilde{\mathbf{G}}_{(4)} \mathbf{G}_{(1)}^{+}+\mathbf{G}_{(2)} \widetilde{\mathbf{G}}_{(3)}^{+} \\
& \quad+\widetilde{\mathbf{G}}_{(3)} \mathbf{G}_{(2)}^{+} .
\end{aligned}
$$

Before finishing this Section, let us note useful interrelations between members of power series for renormalization matrices $\mathbf{C}_{11}$ and $\mathbf{C}_{22}$ on the one hand, and those for delocalization matrices $\mathbf{D}_{(+)}$and $\mathbf{D}_{(-)}$on the other hand. For the first subset, these interrelations are as follows:

$$
\begin{aligned}
& \mathbf{C}_{11}^{(2)}=-\frac{1}{2} \mathbf{D}_{(+)}^{(2)}, \quad \mathbf{C}_{11}^{(3)}=-\frac{1}{2} \mathbf{D}_{(+)}^{(3)}, \\
& \mathbf{C}_{11}^{(4)}=-\frac{1}{2} \mathbf{D}_{(+)}^{(4)}-\frac{1}{2} \mathbf{C}_{11}^{(2)} \mathbf{C}_{11}^{(2)}, \\
& \mathbf{C}_{11}^{(5)}=-\frac{1}{2} \mathbf{D}_{(+)}^{(5)}-\frac{1}{2}\left(\mathbf{C}_{11}^{(2)} \mathbf{C}_{11}^{(3)}+\mathbf{C}_{11}^{(3)} \mathbf{C}_{11}^{(2)}\right),
\end{aligned}
$$

and easily result from the comparison of formulae for $\mathbf{C}_{11}^{(k)}$ (see Eqs. (A23)-(A25)) to those of Eq. (21). Similar relations may be derived for the second subset, too.

\section{Proportionality between populations of basis orbitals and delocalization coefficients of respective localized MOs}

As discussed already, the direct solutions of independent non-canonical one-electron problems (i.e. of the commutation equation and of the block-diagonalization problem) yield expressions for the $\mathrm{CBO}$ matrix $\mathbf{P}$ and for the LMO representation matrix $\mathbf{C}$ in terms of generally different principal submatrices $\mathbf{G}_{(k)}$ and $\widetilde{\mathbf{G}}_{(k)}$, respectively (Appendix A). In this connection, an alternative approach (i.e. the indirect way of obtaining the matrix $\mathbf{P}$ based on employment of one-electron states (MOs) of any type [15, 16, 51]) seems to be more 
promissing when looking for a relation between the actual population of a certain $\mathrm{FO}$ and characteristics of the respective LMO. The most general form of the abovementioned indirect approach consists in constructing a projector [15, 16] to the relevant set of occupied MOs as demonstrated in the Appendix B. The usual definition of the population of a basis function as a sum of increments of all occupied MOs actually is a certain simplified version of the same approach. Just the latter is employed in the present section. Finally, LMOs of Sec. 3 play the role of underlying one-electron states (MOs) in our case.

Let us consider the occupation number (population) $X_{(+) i}$ of an initially-occupied FO $\varphi_{(+) i}$ defined as follows [15, 16]:

$$
X_{(+) i}=2 \sum_{(+) j}^{(\text {OLMOs) }}\left|C_{11, i j}\right|^{2},
$$

where the sum over $(+) j$ embraces now all occupied LMOs (OLMOs) of the system $\left(\psi_{(+) j}\right)$ in contrast to Eqs. (11) and (19), and $C_{11, i j}$ coincides with the respective element of the renormalization matrix $\mathbf{C}_{11}$ (see Eq. (7)). After taking Eqs. (10) and (11) into consideration, the definition of Eq. (23) may be conveniently rewritten as a sum of two increments $A_{i}$ and $B_{i(j)}$, the former representing the contribution of the 'own' occupied LMO of the $i$ th fragment (i.e. of $\psi_{(+) i}$ ) and the latter embracing the increments of all the remaining LMOs $\left(\psi_{(+) j}, j \neq i\right)$. We obtain

$$
X_{(+) i}=A_{i}+B_{i(j)},
$$

where

$$
A_{i}=2\left|C_{11, i i}\right|^{2}, \quad B_{i(j)}=2 \sum_{(+) j(\neq i)}^{(\text {OLMOs })}\left|C_{11, i j}\right|^{2} .
$$

Let us consider these contributions separately.

For further convenience, let us start with the second increment $B_{i(j)}$. The coefficient at the FO $\varphi_{(+) i}$ in the $\mathrm{LMO} \psi_{(+) j}, j \neq i\left(C_{11, i j}\right)$ is expressible in the form of power series,

$$
C_{11, i j}=C_{11, i j}^{(2)}+C_{11, i j}^{(3)}+\ldots
$$

Note that $C_{11, i j}^{(0)}=0$ for $i \neq j$ (see Eq. (10)), whilst the first order member vanishes as $\mathbf{C}_{11}^{(1)}=\mathbf{0}$ (see
Eq. (A13)). The series of Eq. (26) should be now substituted into the second relation of Eq. (25). We obtain

$$
\begin{gathered}
B_{i(j)}=2 \sum_{(+) j(\neq i)}^{(\text {OLMOs })}\left[C_{11, i j}^{(2)} \cdot C_{11, i j}^{(2) *}+C_{11, i j}^{(2)} \cdot C_{11, i j}^{(3) *}\right. \\
\left.\quad+C_{11, i j}^{(3)} \cdot C_{11, i j}^{(2) *}+\ldots\right]
\end{gathered}
$$

where terms to within the fifth order inclusive are explicitly shown and $*$ designates the complex-conjugate counterpart of the respective coefficient. It is seen that the increment $B_{i(j)}$ generally takes a non-zero value. This conclusion may be traced back to tails of occupied LMOs of the intrasubset type (intrasubset delocalization).

Let us turn now to the increment $A_{i}$ containing the diagonal element $\left(C_{11, i i}\right)$ of the same matrix $\left(\mathbf{C}_{11}\right)$. Employment of the power series for this element yields the following expression:

$$
\begin{aligned}
A_{i}=2 & +4 C_{11, i i}^{(2)}+4 C_{11, i i}^{(3)}+4 C_{11, i i}^{(4)}+4 C_{11, i i}^{(5)} \\
& +2\left(C_{11, i i}^{(2)}\right)^{2}+4 C_{11, i i}^{(2)} \cdot C_{11, i i}^{(3)}+\ldots
\end{aligned}
$$

Note that $\mathbf{C}_{11}^{(k)}$ are Hermitian (symmetric) matrices (Appendix A) and thereby $\mathbf{C}_{11, i i}^{(k) *}=\mathbf{C}_{11, i i}^{(k)}$. After invoking the relations of Eq. (22), equation (28) may be reformulated as follows

$$
\begin{aligned}
A_{i}= & -2 D_{(+) i i}^{(2)}-2 D_{(+) i i}^{(3)}-2 D_{(+) i i}^{(4)}-2 D_{(+) i i}^{(5)} \\
& -2\left\{\mathbf{C}_{11}^{(2)} \cdot \mathbf{C}_{11}^{(2)}\right\}_{i i}-2\left\{\mathbf{C}_{11}^{(2)} \cdot \mathbf{C}_{11}^{(3)}\right\}_{i i} \\
& -2\left\{\mathbf{C}_{11}^{(3)} \cdot \mathbf{C}_{11}^{(2)}\right\}_{i i}+2\left(C_{11, i i}^{(2)}\right)^{2} \\
& +4 C_{11, i i}^{(2)} \cdot C_{11, i i}^{(3)}+\ldots
\end{aligned}
$$

It is seen that the contribution of the 'own' LMO $\psi_{(+) i}$ to the actual population $X_{(+) i}$ of the FO $\varphi_{(+) i}$ contains the relevant initial population (2), members of the power series for the total delocalization coefficient of this LMO $\left(D_{(+) i i} \equiv D_{(+) i}\right)$, and terms related to renormalization matrices of lower orders. Moreover, terms of the last type may be represented in a more compact form. Indeed, contributions of the LMO $\psi_{(+) i}$ to $i$ th diagonal elements of products $\mathbf{C}_{11}^{(2)} \cdot \mathbf{C}_{11}^{(2)}, \mathbf{C}_{11}^{(2)} \cdot \mathbf{C}_{11}^{(3)}$, and 
$\mathbf{C}_{11}^{(3)} \cdot \mathbf{C}_{11}^{(2)}$ and the last two terms of the right-hand side of Eq. (29) cancel each other out and we obtain

$$
\begin{aligned}
A_{i}= & 2\left(1-D_{(+) i}\right)-2 \sum_{(+) j(\neq i)}^{(\text {OLMOs })}\left[C_{11, i j}^{(2)} \cdot C_{11, j i}^{(2)}\right. \\
& \left.+C_{11, i j}^{(2)} \cdot C_{11, j i}^{(3)}+C_{11, i j}^{(3)} \cdot C_{11, j i}^{(2)}+\ldots\right] .
\end{aligned}
$$

Now, it remains to sum up the increments $B_{i(j)}$ and $A_{i}$ shown in Eqs. (27) and (30), respectively, in accordance with Eq. (24). It is easily seen that terms of the renormalization origin cancel each other out in the final expression for the occupation number $X_{(+) i}$ (note that $\mathbf{C}_{11, j i}^{(k)}=\mathbf{C}_{11, i j}^{(k) *}$ ). Consequently, sums over occupied LMOs $\psi_{(+) j}, j \neq i$ also vanish in this expression. The result then takes the following simple form:

$$
X_{(+) i}=2\left(1-D_{(+) i}\right) .
$$

Hence, the actual population of the basis function $\varphi_{(+) i}$ of the $i$ th fragment is determined only by the shape of the respective 'own' LMO $\psi_{(+) i}$. Moreover, the population of the $\mathrm{FO} \varphi_{(+) i}$ lost due to the interorbital interaction is proportional to the total intersubset delocalization coefficient of the above-specified exclusive LMO. In other words, the one-to-one correspondence between FOs and LMOs (see Eq. (11)) is now replenished by the following rule: the more delocalized the $\mathrm{FO} \varphi_{(+) i}$ becomes when building up the respective LMO $\psi_{(+) i}$, the more charge it loses and vice versa. Thus, parallelism between charge redistribution and delocalization is proven for each individual pair of electrons. This result may be also interpreted as a kind of simultaneous separability of both charge redistribution and delocalization into increments of individual pairs of electrons. The origin of these principal conclusions easily follows from the above derivation, namely it consists in opposite signs and in coinciding absolute values of terms of the intrasubset nature in the contribution of the 'own' LMO $\psi_{(+) i}$ and in that of the remaining LMOs to the occupation number $X_{(+) i}$. Finally, the relation (31) indicates irrelevance of the intrasubset delocalization in the formation of charge redistribution. This result evidently causes no surprise.

An analogous analysis of the occupation number $X_{(-) m}$ of an initially-vacant basis orbital $\varphi_{(-) m}$ yields the following relation:

$$
X_{(-) m}=2 D_{(-) m} .
$$

Thus, the more delocalized the orbital $\varphi_{(-) m}$ becomes due to interorbital interaction, the more population it actually acquires and vice versa.
Let us dwell now on implications of relations (31) and (32), as well as on their possible extensions. First, generalized matrix forms of these relations may be mentioned that are derived and analysed in the Appendix B (see Eqs. (B5) and (B6)). In this case, the relations embrace the intersubset delocalization matrices $\mathbf{D}_{(+)}$ and $\mathbf{D}_{(-)}$of Eqs. (20) and (21) on the one hand, and the so-called intrasubset population matrices $\mathbf{X}_{(+)}$and $\mathbf{X}_{(-)}$on the other hand, diagonal elements of the latter coinciding with occupation numbers of FOs (i.e. $X_{(+) i} \equiv X_{(+) i i}$ and $\left.X_{(-) m} \equiv X_{(-) m m}\right)$. The second (and the opposite) direction of extending our principal formulae (31) and (32) consists in passing to the relevant partial characteristics. To this end, let us recall the definitions of total delocalization coefficients $D_{(+) i}$ and $D_{(-) m}$ in terms of partial ones shown in Eq. (19) and substitute them into the right-hand sides of Eqs. (31) and (32). As a result, populations $X_{(+) i}$ and $X_{(-) m}$ are expressible as follows:

$$
\begin{aligned}
& X_{(+) i}=2\left(1-\sum_{(-) l}^{(\text {IVFOs })} x_{(+) i,(-) l}\right), \\
& X_{(-) m}=2 \sum_{(+) j}^{(\text {IOFOs })} x_{(-) m,(+) j},
\end{aligned}
$$

where

$$
x_{(+) i,(-) l}=2 d_{(+) i,(-) l}, \quad x_{(-) m,(+) j}=2 d_{(-) m,(+) j}
$$

coincide with the partial population donated by the IOFO $\varphi_{(+) i}$ to the IVFO $\varphi_{(-) l}$ and with that accepted by the IVFO $\varphi_{(-) m}$ from the IOFO $\varphi_{(+) j}$, respectively. Besides, the relation

$$
x_{(+) i,(-) l}=x_{(-) l,(+) i}
$$

is the analogue of Eq. (17) and represents now the charge conservation condition. The results of Eq. (34) show that the more population is transferred between FOs $\varphi_{(+) i}$ and $\varphi_{(-) m}$, the larger is the partial delocalization coefficient of the LMO $\psi_{(+) i}$ over the same IVFO and vice versa. If we recall the definition of this coefficient as a square of the relevant tail of the LMO $\psi_{(+) i}$ (see Eq. (15)), we may also conclude a more significant tail of this LMO over the IVFO $\varphi_{(-) l}$ to correspond to a more efficient charge transfer between FOs $\varphi_{(+) i}$ and $\varphi_{(-) l}$ and vice versa. 
Finally, we may turn to terms of particular order $(k)$ within Eqs. (31), (32), and (34). We then obtain

$$
X_{(+) i}^{(k)}=-2 D_{(+) i}^{(k)}, \quad X_{(-) m}^{(k)}=2 D_{(-) m}^{(k)}
$$

and

$x_{(+) i,(-) l}^{(k)}=2 d_{(+) i,(-) l}^{(k)}, \quad x_{(-) m,(+) j}^{(k)}=2 d_{(-) m,(+) j}^{(k)}$.

The above relations may also be verified directly as described in the Appendix B.

Therefore, parallelism between charge redistribution and delocalization manifests itself at four different levels that are accordingly representable by above-derived proportionalities: (i) between total changes in occupation numbers of FOs and total delocalization coefficients of LMOs (Eqs. (31) and (32)), (ii) between partial populations transferred between orbitals of opposite initial occupation and the relevant partial delocalization coefficients of LMOs (Eq. (34)), as well as between increments of the $k$ th order to (iii) the above-mentioned total characteristics (Eq. (36)) and to (iv) their partial analogues (Eq. (37)). In summary, the overall pattern of the interorbital charge redistribution is predicted to resemble that of delocalization.

\section{The case of first order interorbital interactions. Interpretation of total energies in terms of delocalization of LMOs}

In this section, we will dwell on the practically important case of the above-outlined theory referring to all interorbital interactions of the first order magnitude. In this connection, let us assume $\mathbf{E}_{(+)}$and $\mathbf{E}_{(-)}$of Eqs. (2) to be diagonal matrices, i. e.

$$
E_{(+) i m}=\varepsilon_{(+) i} \delta_{i m}, \quad E_{(-) l r}=\varepsilon_{(-) l} \delta_{l r},
$$

where $\varepsilon_{(+) i}$ and $\varepsilon_{(-) l}$ correspondingly represent oneelectron energies of FOs $\varphi_{(+) i}$ and $\varphi_{(-) l}$. Let us suppose also that our energy reference point is chosen in the middle of the energy gap between IOFOs and IVFOs and the energy unit is a negative quantity in addition. Consequently, $\varepsilon_{(+) i}$ and $\varepsilon_{(-) l}$ are positive parameters, whilst the sum $\varepsilon_{(+) i}+\varepsilon_{(-) l}$ represents the relevant energy gap (the minus sign in front of $\mathbf{E}_{(-)}$of Eq. (2) should also be taken into account here). Two important implications of our principal assumption of Eq. (38) deserve attention in the present context and are discussed below.

First, elements $\left(\widetilde{G}_{(k) i l}\right)$ of matrices $\widetilde{\mathbf{G}}_{(k)}$ are expressible algebraically in this case in terms of those of the first order matrices $\mathbf{S}, \mathbf{Q}$, and $\mathbf{R}$ of Eq. (2) in a close analogy with matrices $\mathbf{G}_{(k)}$ arising in the CBO matrix $\mathbf{P}$ [10]. Let the above-specified elements $S_{i j}, R_{i l}$, and $Q_{l r}$ be represented as follows:

$$
S_{i j}=\left\langle\varphi_{(+) i}|\widehat{H}| \varphi_{(+) j}\right\rangle, R_{i l}=\left\langle\varphi_{(+) i}|\widehat{H}| \varphi_{(-) l}\right\rangle,
$$

$Q_{l r}=\left\langle\varphi_{(-) l}|\widehat{H}| \varphi_{(-) r}\right\rangle$,

where the respective basis orbitals (FOs) are indicated inside the bra- and ket-vectors. As already mentioned (Sec. 2), the first two members of the series $\widetilde{\mathbf{G}}_{(k)}, k=$ $1,2, \ldots$ coincide with those of the former series $\mathbf{G}_{(k)}$, $k=1,2, \ldots$, i.e. $\widetilde{\mathbf{G}}_{(1)}=\mathbf{G}_{(1)}$ and $\widetilde{\mathbf{G}}_{(2)}=\mathbf{G}_{(2)}$. The same coincidence evidently refers to the relevant elements. Thus, the expressions of Ref. [10] may be invoked here along with respective interpretations. For instance, the first order element $G_{(1) i l}$ takes the form

$$
G_{(1) i l}=-\frac{R_{i l}}{\varepsilon_{(+) i}+\varepsilon_{(-) l}}
$$

and describes the direct interaction between basis orbitals $\varphi_{(+) i}$ and $\varphi_{(-) l}$. The second order element $G_{(2) i l}$, in turn, is expressible as follows:

$$
\begin{aligned}
G_{(2) i l}= & \frac{1}{\varepsilon_{(+) i}+\varepsilon_{(-) l}}\left\{\sum_{(+) j}^{(\mathrm{IOFOs})} \frac{S_{i j} R_{j l}}{\varepsilon_{(+) j}+\varepsilon_{(-) l}}\right. \\
& \left.-\sum_{(-) r}^{(\text {IVFOs })} \frac{R_{i r} Q_{r l}}{\varepsilon_{(+) i}+\varepsilon_{(-) r}}\right\} .
\end{aligned}
$$

This element represents the indirect interaction between the same orbitals $\left(\varphi_{(+) i}\right.$ and $\left.\varphi_{(-) l}\right)$ by means of a single mediator. Both IOFOs $\left(\varphi_{(+) j}\right)$ and IVFOs $\left(\varphi_{(-) r}\right)$ are able to play this role. To be an efficient mediator, however, the orbitals concerned should interact directly with both $\varphi_{(+) i}$ and $\varphi_{(-) l}$.

As opposed to the above-considered first and second order elements, the expression for $\widetilde{G}_{(3) i l}$ does not coincide with that for $G_{(3) i l}$ (Appendix A). Nevertheless, it may be easily derived on the basis of Eq. (A28) after employment of Eq. (A21). Moreover, the element $\widetilde{G}_{(3) i l}$ may be shown to describe an indirect interaction between FOs $\varphi_{(+) i}$ and $\varphi_{(-) l}$ by means of two mediators as it was the case with $G_{(3) i l}$ [52, 53]. An analogous state of things refers to elements of matrices $\widetilde{\mathbf{G}}_{(k)}$ of higher orders too. Let us recall now that the tail of the occupied LMO $\psi_{(+) i}$ over the IVFO $\varphi_{(-) l}$ is determined by the sum over the order parameter $k$ of elements $\widetilde{G}_{(k) i l}$ (see Eqs. (11) and (14)). The same 
refers also to the relevant partial delocalization coefficient $d_{(+) i,(-) l}$ defined by Eq. (15). We may conclude on this basis that both the coefficient $d_{(+) i,(-) l}$ and the above-specified tail depend on the sum of all possible (direct and indirect) interactions between orbitals $\varphi_{(+) i}$ and $\varphi_{(-) l}$. The non-local nature of matrix elements $G_{(2) i l}, \widetilde{G}_{(3) i l}, \widetilde{G}_{(4) i l}$, etc also deserves mentioning, and the expression of Eq. (41) illustrates this statement (the first order element $G_{(1) i l}$ makes an exception here). Hence, the both partial characteristics $d_{(+) i,(-) l}$ and $x_{(+) i,(-) l}$ also are non-local in their nature.

The second important implication of Eq. (38) consists in the possibility of expressing each member of the power series $\left(\mathcal{E}_{(k)}\right)$ for the total energy of our system $(\mathcal{E})$ in terms of contributions of the same order $\left(d_{(+) i,(-) l}^{(k)}\right)$ to partial delocalization coefficients $d_{(+) i,(-) l}$. The simplest way of performing this procedure consists in employment of the results of Ref. [11]. Indeed, the above-specified increments $\mathcal{E}_{(k)}, k \geqslant 2$ concerning any system described by the matrix $\mathbf{H}$ of Eqs. (1) and (2) have been expressed in this study in terms of partial populations of the same order $\left(x_{(+) i,(-) l}^{(k)}\right)$ transferred between orbitals (FOs) of opposite initial occupation, namely,

$$
\mathcal{E}_{(k)}=\frac{1}{k-1} \sum_{(+) i}^{(\mathrm{IOFOs})} \sum_{(-) l}^{(\mathrm{IVFOs})} x_{(+) i,(-) l}^{(k)}\left(\varepsilon_{(+) i}+\varepsilon_{(-) l}\right),
$$

Besides, the sum of the initial members of the same series (i. e. $\mathcal{E}_{(0)}+\mathcal{E}_{(1)}$ ) coincides with the total energy of isolated IOFOs (Appendix C). The expression of Eq. (42) indicates that the more population is generally transferred between IOFOs and IVFOs, the more stabilized the whole system becomes versus the set of isolated IOFOs and vice versa. It also deserves adding here that Eq. (42) has been obtained using the CBO matrix $\mathbf{P}$, the latter resulting from solution of the commutation equation.

If we recall now the above-established proportionality between $x_{(+) i,(-) l}^{(k)}$ and $d_{(+) i,(-) l}^{(k)}$ shown in Eq. (37), we obtain an alternative form of $\mathcal{E}_{(k)}$ in terms of coefficients $d_{(+) i,(-) l}^{(k)}$, i. e.,

$\mathcal{E}_{(k)}=\frac{2}{k-1} \sum_{(+) i}^{(\text {OLMOs) }} \sum_{(-) l}^{(\text {IVFOs })} d_{(+) i,(-) l}^{(k)}\left(\varepsilon_{(+) i}+\varepsilon_{(-) l}\right)$,

where $d_{(+) i,(-) l}^{(k)}$ are shown in Eq. (18).
An alternative way of deriving Eq. (43) lies in employment of the expression for $\mathcal{E}_{(k)}$ in terms of intersubset delocalization matrices $\mathbf{D}_{(+)}$and $\mathbf{D}_{(-)}$of Eq. (C12) (Appendix C). As opposed to Eq. (42), the relation $(\mathrm{C} 12)$ results from the solution of the blockdiagonalization problem for our Hamiltonian matrix (Sec. 2). After an additional invoking of Eq. (38), we obtain

$$
\begin{aligned}
\mathcal{E}_{(k)}= & \frac{2}{k-1}\left[\sum_{(+) i}^{(\text {OLMOs })} D_{(+) i i}^{(k)} \varepsilon_{(+) i}\right. \\
& \left.+\sum_{(-) l}^{\text {(VLMOs) }} D_{(-) l l}^{(k)} \varepsilon_{(-) l}\right]
\end{aligned}
$$

where $D_{(+) i i}^{(k)} \equiv D_{(+) i}^{(k)}$ and $D_{(-) l l}^{(k)} \equiv D_{(-) l}^{(k)}$ coincide with total delocalization coefficients of LMOs $\psi_{(+) i}$ and $\psi_{(-) l}$ defined by Eq. (19). Substituting (19) into (44) followed by invoking Eq. (17) yields Eq. (43) in accordance with the expectation.

It is seen, therefore, that the charge transfer energy of Ref. [11] is actually equivalent to the energy of the intersubset delocalization of occupied LMOs over IVFOs. Moreover, stabilization (or destabilization) of our system due to interorbital interaction proves to be directly related to the extents of delocalization of the initiallylocalized pairs of electrons. This principal conclusion causes little surprise if we recall popular classical assumptions about an interdependence between stabilization and delocalization (Sec. 1).

\section{Illustration of the results by consideration of specific reactions}

The principal relations of the present study shown in Eqs. (31), (32), and (34)-(37) along with the abovedemonstrated equivalence of Eqs. (42) and (43) provide us with an efficient tool for a new interpretation of organic reactions. Indeed, let a certain route of reaction be characterized by a more efficient charge transfer from the IOFO $\varphi_{(+) i}$ of the $i$ th fragment to the IVFO $\varphi_{(-) l}$ of the $l$ th one versus the alternative routes as it was the case with numerous specific processes studied previously [7-9] and discussed below in this section. We may then expect a more significant tail of the LMO $\psi_{(+) i}$ to arise over the same IVFO. The relevant initially-localized pair of electrons of the $i$ th fragment is accordingly predicted to be delocalized more efficiently over the $l$ th one. Thus, an interpretation of the reaction in terms of variable extents of delocalization 
of separate pairs of electrons actually follows. Inasmuch as more efficient charge redistributions between FOs of the relevant reaction centre and of its nearest neighbourhood [8] were shown to be peculiar to predominant (allowed [9]) routes of reactions, just these exclusive routes may be anticipated to be characterized by an enhanced delocalization of the principal pairs of electrons.

To verify and to illustrate these general predictions, let us turn now to specific examples. Let us start with the well-known bimolecular nucleophilic substitution $\left(\mathrm{S}_{N} 2\right)$ process between a substituted alkane $\mathrm{Z}-\mathrm{C}_{\alpha} \mathrm{H}_{2}-$ $\mathrm{C}_{\beta} \mathrm{H}_{2}-\ldots$ and nucleophile $(N u)[1-6,54,55]$, where $\mathrm{Z}$ stands for a heteroatom (nucleofuge). A back attack of the reagent and not a frontal one is commonly assumed to give rise to a subsequent substitution process. These routes of reaction have been correspondingly considered as allowed and forbidden ones [9]. In respect of efficiencies of charge redistribution between FOs, comparison of the same alternatives has been made in Refs. [8, 9, 56]. To this end, the interactions (resonance parameters) between the principal electron-donating orbital of nucleophile $\left(\varphi_{(+) N}\right)$ and the electron-accepting (antibonding) orbital of the $\mathrm{C}_{\alpha}-\mathrm{Z}$ bond $\left(\varphi_{(-) a}\right)$ were assumed to take coinciding absolute values for both positions of the reagent (similar values of these parameters are expected on the basis of the relevant estimations [56]). Consequently, the second order contributions $\left(x_{(+) N,(-) a}^{(2)}\right)$ to respective partial populations $\left(x_{(+) N,(-) a}\right)$ are uniform for both attacks, i. e.,

$$
x_{(+) N,(-) a}^{(2) \text { back }}=x_{(+) N,(-) a}^{(2) \text { front }}>0 .
$$

(Positive signs of these contributions follow directly from Eqs. (18) and (37).) Meanwhile, the relevant third order increments were shown to be of opposite signs,

$$
x_{(+) N,(-) a}^{(3) \text { back }}>0, \quad x_{(+) N,(-) a}^{(3) \text { front }}<0 .
$$

When added to their second order counterparts of Eq. (45), the corrections of Eq. (46) ensure a more efficient partial charge transfer between orbitals $\varphi_{(+) N}$ and $\varphi_{(-) a}$ for the back attack (versus the frontal one) and thereby a larger value of the relevant increment to the overall stabilization energy (see Eq. (42)). After employment of Eq. (37), we accordingly obtain

$$
d_{(+) N,(-) a}^{(2) \text { back }}=d_{(+) N,(-) a}^{(2) \text { front }}>0
$$

and

$$
d_{(+) N,(-) a}^{(3) \mathrm{back}}>0, \quad d_{(+) N,(-) a}^{(3) \text { front }}<0 .
$$

The above relations indicate a more significant partial delocalization coefficient $d_{(+) N,(-) a}$ to refer to the predominant (allowed) back attack of nucleophile. In other words, better conditions are ensured for delocalization of the lone pair of electrons of nucleophile over the reacting $\mathrm{C}_{\alpha}-\mathrm{Z}$ bond, if the reagent takes the back position with respect to the reactant. Just this fact may be concluded to determine the enhanced reactivity in this case. The above conclusions are entirely based on analysis of third order terms of power series derived in the present study.

The aromatic electrophilic substitution $\left(\mathrm{S}_{E} 2\right)$ reaction of pyridine [55, 57-59] may be discussed here as another example of processes governed by third order terms [9]. Exclusively the meta-substituted pyridines are known to result from these reactions in contrast to uniform reactivities of all positions of benzene. Moreover, even the most reactive meta-positioned carbon atoms of the heterocycle are considerably less reactive as compared to particular atoms of the parent hydrocarbon. In respect of charge redistributions, this process has been analysed in Ref. [60]. The usual (canonical) MOs of benzene played the role of FOs in this study along with a single IVFO of electrophile $\left(\varphi_{(-) E}\right)$. The third order member $\left(x_{(+) 2,(-) E}^{(3)}\right)$ of the power series for the partial population $x_{(+) 2,(-) E}$ transferred between the principal electron-donating (initially-occupied) orbital of the reactant $\left(\varphi_{(+) 2}\right)$ and that of electrophile $\left(\varphi_{(-) E}\right)$ was shown to be responsible for the alteration in the relative reactivity after introducing the nitrogen atom. [The orbital $\varphi_{(+) 2}$ coincides with the HOMO of benzene of the appropriate symmetry (symmetric relatively to the plane embracing the first and the fourth carbon atoms, the former corresponding to the site of the nitrogen atom).] Furthermore, analysis of expressions for corrections $x_{(+) 2,(-) E}^{(3)}$ showed them to be negative quantities, whatever the direction of the attack. Using Eq. (37) we may then conclude that the suppressed overall reactivity of pyridine versus that of benzene may be traced back to worse conditions for delocalization of the principal pair of electrons of the reactant over the orbital of the approaching reagent in the case of the heterocycle. So far as different reactivities of separate carbon atoms of pyridine are concerned, these have been accounted for in Ref. [60] by the smallest absolute value of the correction $x_{(+) 2,(-) E}^{(3) m e t a}$ as compared to those of $x_{(+) 2,(-) E}^{(3) \text { para }}$ and $x_{(+) 2,(-) E}^{(3) \text { ortho }}$. In terms of delocalization, this implies somewhat better conditions for this effect in the case of meta-oriented process as compared to the remaining alternatives. 
Organic reactions governed by corrections of power series of higher orders ( $k=4$ and 5) [9] may be interpreted similarly. To avoid a lengthy discussion, let us confine ourselves to an overview of final conclusions.

Let us consider the bimolecular electrophilic addition $\left(\operatorname{Ad}_{E} 2\right)$ process of substituted ethenes $\mathrm{H}_{2} \mathrm{C}_{\beta}=\mathrm{C}_{\alpha} \mathrm{HZ}$ analysed in Ref. [7], where $\mathrm{Z}$ stands for a substituent. The alternative routes under comparison coincide now with additions to $\mathrm{C}_{\alpha}$ and $\mathrm{C}_{\beta}$ atoms (the so-called $\alpha$-and $\beta$-additions). For an electron-donating substituent $\mathrm{Z}=\mathrm{D}$, the $\beta$-attack is known to be of considerably greater reactivity versus the $\alpha$-attack (see the Markovnikov rule [1-4, 6]). In terms of delocalization of LMOs, these trends may be interpreted as follows: the lone pair of electrons of the substituent $\mathrm{D}$ becomes delocalized more significantly both over the antibonding orbital of the ethene fragment $(\mathrm{C}=\mathrm{C}$ bond $)$ and over the IVFO of electrophile, if the reagent approaches the $\mathrm{C}_{\beta}$ atom versus the $\mathrm{C}_{\alpha}$ atom. Similarly, the initiallylocalized pair of electrons of the $\mathrm{C}=\mathrm{C}$ bond proves to be delocalized more substantially over the IVFO of electrophile in the case of the $\beta$-attack. On the whole, these results imply a kind of an enhanced attractive effect of electrophile upon both pairs of electrons of the reactant in the case of the predominant (allowed [9]) $\beta$-attack versus the alternative (forbidden) one. In the case of an electron-accepting substituent $(\mathrm{Z}=\mathrm{A})$, the $\mathrm{C}_{\alpha}$ atom is known to be more reactive towards electrophiles as compared to the $\mathrm{C}_{\beta}$ atom [2, 3, 6]. Analogously, the pair of electrons of the $\mathrm{C}=\mathrm{C}$ bond becomes delocalized more substantially over the IVFO of electrophile just for the $\alpha$-attack.

Furthermore, the trans $\beta$-elimination is known to predominate over the relevant cis process in the concerted bimolecular elimination (E2) reactions of substituted alkanes [2, 3, 5]. Analysis of electron density redistributions among orbitals of separate bonds of the reactant and of an external base has been performed in Ref. [61]. Terms of the fifth order $(k=5)$ were shown to play the decisive role in this case. Invoking these results along with the relation (37) shows that both the lone pair of electrons of the base and that of the $\mathrm{H}-\mathrm{C}_{\beta}$ bond become delocalized over the antibonding orbital of the $\mathrm{Z}-\mathrm{C}_{\alpha}$ bond more significantly just for the trans elimination as compared to the relevant cis process.

Finally, pericyclic reactions may also be considered similarly. For illustration, let us refer to the thermal electrocyclic closure of polyenes and to the Diels-Alder reactions studied by means of the semilocalized approach in Refs. [53, 62]. More significant electron density redistributions among initially-occupied and initially-vacant orbitals of both first-neighbouring and second-neighbouring $\mathrm{C}=\mathrm{C}$ bonds were shown to be peculiar to allowed routes of these processes versus the forbidden ones. On the basis of the present results, just the former (predominant) routes may be concluded to be accompanied by better conditions for delocalization of pairs of electrons of $\mathrm{C}=\mathrm{C}$ bonds over antibonding orbitals of neighbouring bonds.

Therefore, the expectation about predominant (allowed) routes of organic reactions to be characterized by enhanced delocalization of the principal pairs of electrons appears to be supported by analysis of specific examples.

\section{Conclusions}

1. The principal achievement of the above study consists in establishing direct proportionalities between characteristics of charge redistribution due to chemical interaction on the one hand, and their counterparts representing the extents of delocalization of separate pairs of electrons on the other hand. Moreover, the total energy of the system is shown to be alternatively expressible either in terms of interorbital charge transfer or via delocalization coefficients of LMOs. This implies charge redistribution and delocalization to be interdependent effects or even to represent different aspects of the same phenomenon.

2. The population lost by a certain initially-doublyoccupied basis orbital (FO) due to interorbital interaction is shown to be determined by reshaping of only a single LMO. Thus, a one-orbital representation of the lost population actually follows from the non-canonical method of MOs. This state of things closely resembles the one-orbital representation of ionization potentials in the canonical MO method known as the Koopmans' theorem (see e.g. [16, 21]). If we recall that representations of this type are not achievable for ionization potentials and for charge redistributions in the NCMO and the CMO methods respectively, the present results support the complementary nature of the above-mentioned principal approaches of quantum chemistry [63].

3. The results of the above study demonstrate separability of the overall charge redistribution into increments of individual pairs of electrons in the space of localized basis functions (FOs). In this respect, the present approach may be regarded as a quantum-chemical analogue of the classical "curly 
arrow chemistry'. In contrast to the latter, however, the approach suggested allows comparisons of relative extents of shifts of separate pairs of electrons for alternative routes of the given reaction.

4. Predominant (allowed) and alternative (forbidden) routes of both heterolytic and pericyclic organic reactions are shown to be correspondingly characterized by enhanced and suppressed delocalization of the principal pairs of electrons. This implies better conditions for delocalization of initiallylocalized pairs of electrons to be the driving force of the above-mentioned processes.

5. The results obtained give us a new insight into the nature of local models of organic reactions. In particular, confinement to a local set of basis functions (FOs) of a certain fragment (e.g. of the reaction centre) actually implies taking into account only a limited number of semi-localized pairs of electrons, namely of those attached to the given fragment of the whole system. Reshaping of these pairs due to intermolecular chemical interaction, however, is generally governed by constitution of the nearest neighbourhood of the principal fragment, too.

\section{Appendix A}

\section{Solution of the block-diagonalization problem}

Let us dwell here on the block-diagonalization problem shown in Eqs. (4) and/or (5) and referring to the Hamiltonian matrix defined by Eqs. (1) and (2). The additional unitarity requirement for the LMO representation matrix (C) being sought is shown in Eq. (3).

As already mentioned (Sec. 2), the matrix $\mathbf{C}$ is representable in the form of power series (see Eq. (9)). Let us now substitute this series into Eqs. (3) and (4). Collecting terms of the same order $(k)$ then yields the following relations:

$$
\begin{gathered}
\mathbf{C}_{(1)}^{+}+\mathbf{C}_{(1)}=\mathbf{0} \\
\mathbf{C}_{(2)}^{+}+\mathbf{C}_{(2)}+\mathbf{C}_{(1)}^{+} \mathbf{C}_{(1)}=\mathbf{0} \\
\mathbf{C}_{(3)}^{+}+\mathbf{C}_{(3)}+\mathbf{C}_{(1)}^{+} \mathbf{C}_{(2)}+\mathbf{C}_{(2)}^{+} \mathbf{C}_{(1)}=\mathbf{0}, \\
\mathbf{C}_{(4)}^{+}+\mathbf{C}_{(4)}+\mathbf{C}_{(1)}^{+} \mathbf{C}_{(3)}+\mathbf{C}_{(3)}^{+} \mathbf{C}_{(1)}+\mathbf{C}_{(2)}^{+} \mathbf{C}_{(2)}=\mathbf{0},
\end{gathered}
$$

etc. Similarly, from Eq. (4) we obtain

$$
\left\{\mathbf{C}_{(1)}^{+} \mathbf{H}_{(0)}+\mathbf{H}_{(0)} \mathbf{C}_{(1)}+\mathbf{H}_{(1)}\right\}_{12}=\mathbf{0},
$$

$$
\begin{aligned}
& \left\{\mathbf{C}_{(2)}^{+} \mathbf{H}_{(0)}+\mathbf{H}_{(0)} \mathbf{C}_{(2)}+\mathbf{C}_{(1)}^{+} \mathbf{H}_{(0)} \mathbf{C}_{(1)}+\mathbf{C}_{(1)}^{+} \mathbf{H}_{(1)}\right. \\
& \left.+\mathbf{H}_{(1)} \mathbf{C}_{(1)}\right\}_{12}=\mathbf{0}
\end{aligned}
$$

etc, along with analogous conditions for the remaining off-diagonal block 21. Let us now express $\mathbf{C}_{(k)}^{+}$via $\mathbf{C}_{(k)}$ on the basis of Eqs. (A1)-(A4) and substitute the resulting formulae into Eqs. (A5), (A6), etc to eliminate $\mathbf{C}_{(k)}^{+}$ from the latter. The general form of relations obtained is as follows:

$$
\begin{gathered}
\left\{\left[\mathbf{H}_{(0)}, \mathbf{C}_{(k)}\right]_{-}+\mathbf{U}_{(k)}\right\}_{12}= \\
\left\{\left[\mathbf{H}_{(0)}, \mathbf{C}_{(k)}\right]_{-}+\mathbf{U}_{(k)}\right\}_{21}=\mathbf{0},
\end{gathered}
$$

where

$$
\begin{aligned}
\mathbf{U}_{(1)}= & \mathbf{H}_{(1)}, \\
\mathbf{U}_{(2)}= & {\left[\mathbf{H}_{(1)}, \mathbf{C}_{(1)}\right]_{-}+\mathbf{C}_{(1)}^{+} \mathbf{H}_{(0)} \mathbf{C}_{(1)} } \\
& -\mathbf{C}_{(1)}^{+} \mathbf{C}_{(1)} \mathbf{H}_{(0)},
\end{aligned}
$$

and the notation $[\ldots, \ldots]_{-}$stands for the commutator of matrices.

Let us now invoke the expressions for corrections $\mathbf{C}_{(k)}$ in terms of four blocks $\mathbf{C}_{I J}^{(k)}, I, J=1,2$ (see Eqs. (7) and (10)). From Eq. (A7) we then obtain the following matrix equations:

$$
\begin{array}{r}
\mathbf{E}_{(+)} \mathbf{C}_{12}^{(1)}+\mathbf{C}_{12}^{(1)} \mathbf{E}_{(-)}+\mathbf{R}=\mathbf{0}, \\
-\mathbf{E}_{(-)} \mathbf{C}_{21}^{(1)}-\mathbf{C}_{21}^{(1)} \mathbf{E}_{(+)}+\mathbf{R}^{+}=\mathbf{0},
\end{array}
$$

determining the off-diagonal blocks of the first order correction $\mathbf{C}_{(1)}$, i.e. $\mathbf{C}_{12}^{(1)}$ and $\mathbf{C}_{21}^{(1)}$. As in our previous studies [7-11, 19, 48, 49, 52, 53, 56, 60-62], let $\mathbf{C}_{12}^{(1)}$ be denoted by $\mathbf{G}_{(1)}$. The principal equation conditioning the latter then takes the form

$$
\mathbf{E}_{(+)} \mathbf{G}_{(1)}+\mathbf{G}_{(1)} \mathbf{E}_{(-)}+\mathbf{R}=\mathbf{0},
$$

whereas $\mathbf{C}_{21}^{(1)}$ coincides with $-\mathbf{G}_{(1)}^{+}$as Eq. (A10) indicates. Moreover, zero diagonal blocks $\mathbf{C}_{11}^{(1)}$ and $\mathbf{C}_{22}^{(1)}$ follow from Eq. (A1), provided that Hermitian (symmetric) nature of these blocks is additionally assumed, i. e.,

$$
\mathbf{C}_{11}^{(1)}=\mathbf{C}_{11}^{(1)+}=\mathbf{0}, \quad \mathbf{C}_{22}^{(1)}=\mathbf{C}_{22}^{(1)+}=\mathbf{0} .
$$


In summary, the total first order member of the power series for the LMO representation matrix takes the following anti-block-diagonal form:

$$
\mathbf{C}_{(1)}=\left|\begin{array}{cc}
\mathbf{0} & \mathbf{G}_{(1)} \\
-\mathbf{G}_{(1)}^{+} & \mathbf{0}
\end{array}\right|
$$

The second order correction $\mathbf{C}_{12}^{(2)}=\mathbf{G}_{(2)}$ may be derived analogously. We obtain

$$
\mathbf{E}_{(+)} \mathbf{G}_{(2)}+\mathbf{G}_{(2)} \mathbf{E}_{(-)}+\mathbf{V}_{(2)}=\mathbf{0}
$$

instead of Eq. (A11), where

$$
\mathbf{V}_{(2)}=\mathbf{S G}_{(1)}-\mathbf{G}_{(1)} \mathbf{Q} .
$$

Moreover, the relation $\mathbf{C}_{21}^{(2)}=-\mathbf{G}_{(2)}^{+}$also easily results from Eq. (A7). The diagonal blocks of the second order correction (i.e. $\mathbf{C}_{11}^{(2)}$ and $\mathbf{C}_{22}^{(2)}$ ), in turn, follow from Eq. (A2) and are non-zero matrices in contrast to their first order counterparts. The total second order member $\mathbf{C}_{(2)}$ of the power series concerned takes then the form

$$
\mathbf{C}_{(2)}=\left|\begin{array}{cc}
-\frac{1}{2} \mathbf{G}_{(1)} \mathbf{G}_{(1)}^{+} & \mathbf{G}_{(2)} \\
-\mathbf{G}_{(2)}^{+} & -\frac{1}{2} \mathbf{G}_{(1)}^{+} \mathbf{G}_{(1)}
\end{array}\right| .
$$

The above formulae have been originally derived in Ref. [10]. As already mentioned (Sec. 1), corrections $\mathbf{C}_{(1)}$ and $\mathbf{C}_{(2)}$ of Eqs. (A13) and (A16) closely resemble the respective members of the power series for the CBO matrix $\mathbf{P}$, i. e. $\mathbf{P}_{(1)}$ and $\mathbf{P}_{(2)}$. The latter have been derived in Ref. [10] on the basis of a direct solution of the so-called commutation equation for the one-electron DM and contain the same matrices $\mathbf{G}_{(1)}$ and $\mathbf{G}_{(2)}$ in analogous positions,

$$
\begin{aligned}
& \mathbf{P}_{(1)}=-2\left|\begin{array}{cc}
\mathbf{0} & \mathbf{G}_{(1)} \\
\mathbf{G}_{(1)}^{+} & \mathbf{0}
\end{array}\right|, \\
& \mathbf{P}_{(2)}=-2\left|\begin{array}{cc}
\mathbf{G}_{(1)} \mathbf{G}_{(1)}^{+} & \mathbf{G}_{(2)} \\
\mathbf{G}_{(2)}^{+} & -\mathbf{G}_{(1)}^{+} \mathbf{G}_{(1)}
\end{array}\right| .
\end{aligned}
$$

Common structures of matrices $\mathbf{C}_{(1)}$ and $\mathbf{P}_{(1)}$, as well as of $\mathbf{C}_{(2)}$ and $\mathbf{P}_{(2)}$ played the decisive role in establishing direct proportionalities between occupation numbers of FOs and delocalization coefficients of LMOs to within $k=2$ inclusive [10, 42]. For higher values of the order parameter $k$, however, the extent of similarity between corrections $\mathbf{C}_{(k)}$ and $\mathbf{P}_{(k)}$ become significantly lower. Let us discuss this important point in more detail.

The above-mentioned close resemblance between $\mathbf{C}_{(1)}$ and $\mathbf{P}_{(1)}$, as well as between $\mathbf{C}_{(2)}$ and $\mathbf{P}_{(2)}$, was based on the fact that the commutation equation for the DM also yields intermediate relations like those of Eq. (A7), where the matrices

$$
\mathbf{W}_{(k)}=\left[\mathbf{H}_{(1)}, \mathbf{P}_{(k-1)}\right]_{-}
$$

stand instead of $\mathbf{U}_{(k)}$. Hence, the extent of similarity between $\mathbf{C}_{(k)}$ and $\mathbf{P}_{(k)}$ generally depends on that between the off-diagonal blocks of matrices $\mathbf{U}_{(k)}$ and $\mathbf{W}_{(k)}$ for the given value of $k$. For $k=1$ and $k=2$, these blocks were shown to be mutually proportional. [In the case of $k=2$, contributions of the second and third terms of the matrix $\mathbf{U}_{(2)}$ of Eq. (A8) to the above-mentioned off-diagonal blocks vanish, and the matrix $\mathbf{U}_{(2)}$ may be actually replaced by the commutator $\left[\mathbf{H}_{(1)}, \mathbf{C}_{(1)}\right]_{-}$, the latter being similar to the matrix $\mathbf{W}_{(2)}$ as Eq. (A18) indicates. That is why the relevant corrections $\mathbf{C}_{(2)}$ and $\mathbf{P}_{(2)}$ closely resemble each other.] For $k=3$, 4, etc, however, there is no direct proportionality between the off-diagonal blocks of matrices $\mathbf{U}_{(k)}$ and $\mathbf{W}_{(k)}$.

Let us return again to our block-diagonalization problem and consider the third order members as an example. An intermediate equation like that of Eq. (A7) may be derived for $k=3$ as well, where

$$
\begin{aligned}
& \mathbf{U}_{(3)}=\left[\mathbf{H}_{(1)}, \mathbf{C}_{(2)}\right]_{-} \\
& +\mathbf{C}_{(1)}^{+}\left\{\left[\mathbf{H}_{(0)}, \mathbf{C}_{(2)}\right]_{-}+\left[\mathbf{H}_{(1)}, \mathbf{C}_{(1)}\right]_{-}\right\} \\
& -\left(\mathbf{C}_{(2)}+\mathbf{C}_{(1)}^{+} \mathbf{C}_{(1)}\right)\left[\mathbf{H}_{(0)} \cdot \mathbf{C}_{(1)}\right]_{-} .
\end{aligned}
$$

As opposed to $\mathbf{U}_{(2)}$ of Eq. (A8), the above-exhibited matrix $\mathbf{U}_{(3)}$ cannot be replaced by the first term $\left[\mathbf{H}_{(1)}, \mathbf{C}_{(2)}\right]_{-}$, because the remaining terms of Eq. (A19) also contribute to its off-diagonal blocks $\mathbf{U}_{12}^{(3)}$ and $\mathbf{U}_{21}^{(3)}$. Consequently, a new matrix $\widetilde{\mathbf{G}}_{(3)}$ is now obtained that serves to express the off-diagonal blocks of the correction $\mathbf{C}_{(3)}$ :

$$
\mathbf{C}_{12}^{(3)}=\widetilde{\mathbf{G}}_{(3)}, \quad \mathbf{C}_{21}^{(3)}=-\widetilde{\mathbf{G}}_{(3)}^{+} .
$$

Equation conditioning the matrix $\widetilde{\mathbf{G}}_{(3)}$ resembles that of Eq. (A14), where the analogue of the matrix $\mathbf{V}_{(2)}$ takes the form

$$
\begin{aligned}
& \widetilde{\mathbf{V}}_{(3)}=\mathbf{S G}_{(2)}-\mathbf{G}_{(2)} \mathbf{Q} \\
& -\frac{1}{2}\left(\mathbf{R G}_{(1)}^{+} \mathbf{G}_{(1)}+\mathbf{G}_{(1)} \mathbf{G}_{(1)}^{+} \mathbf{R}+\mathbf{G}_{(1)} \mathbf{R}^{+} \mathbf{G}_{(1)}\right) .
\end{aligned}
$$

Distinction between matrices $\widetilde{\mathbf{G}}_{(3)}$ and $\mathbf{G}_{(3)}$ (the latter taking the off-diagonal positions in the correction $\mathbf{P}_{(3)}$ 
of the CBO matrix $\mathbf{P}$ ) deserves an additional emphasizing here. Indeed, the term $\mathbf{V}_{(3)}$ conditioning the matrix $\mathbf{G}_{(3)}$ is [11]

$$
\mathbf{V}_{(3)}=\mathbf{S G}_{(2)}-\mathbf{G}_{(2)} \mathbf{Q}-\left(\mathbf{R G}_{(1)}^{+} \mathbf{G}_{(1)}+\mathbf{G}_{(1)} \mathbf{G}_{(1)}^{+} \mathbf{R}\right)
$$

and does not coincide with $\widetilde{\mathbf{V}}_{(3)}$ of Eq. (A21). Consequently, dissimilar off-diagonal blocks prove to be peculiar to corrections $\mathbf{C}_{(3)}$ and $\mathbf{P}_{(3)}$. Meanwhile, the relevant diagonal blocks remain mutually proportional. From Eq. (A3), we obtain

$$
\begin{aligned}
& \mathbf{C}_{11}^{(3)}=-\frac{1}{2}\left(\mathbf{G}_{(1)} \mathbf{G}_{(2)}^{+}+\mathbf{G}_{(2)} \mathbf{G}_{(1)}^{+}\right), \\
& \mathbf{C}_{22}^{(3)}=-\frac{1}{2}\left(\mathbf{G}_{(1)}^{+} \mathbf{G}_{(2)}+\mathbf{G}_{(2)}^{+} \mathbf{G}_{(1)}\right) .
\end{aligned}
$$

The same procedure may be easily performed also for higher values of the order parameter $k$. For $k \geqslant 4$, however, dissimilarity between submatrices of corrections $\mathbf{C}_{(k)}$ and $\mathbf{P}_{(k)}$ embraces both diagonal and off-diagonal positions. Thus, the overall distinction between members of power series concerned grows under increasing values of the order parameter. To achieve the ends of the present study, we may confine ourselves to diagonal submatrices of corrections $\mathbf{C}_{(4)}$ and $\mathbf{C}_{(5)}$. For $k=4$, the relevant formulae follow from Eq. (A4), where the product $\mathbf{C}_{(2)}^{+} \mathbf{C}_{(2)}$ plays an important role. Indeed, just this term yields renormalization matrices of the second order in the final expressions for $\mathbf{C}_{11}^{(4)}$ and $\mathbf{C}_{22}^{(4)}$. For instance, we obtain

$$
\begin{aligned}
& \mathbf{C}_{11}^{(4)}=\mathbf{C}_{11}^{(4)+}= \\
& -\frac{1}{2}\left(\mathbf{G}_{(1)} \widetilde{\mathbf{G}}_{(3)}^{+}+\widetilde{\mathbf{G}}_{(3)} \mathbf{G}_{(1)}^{+}+\mathbf{G}_{(2)} \mathbf{G}_{(2)}^{+}\right) \\
& -\frac{1}{2} \mathbf{C}_{11}^{(2)} \mathbf{C}_{11}^{(2)} .
\end{aligned}
$$

The fifth order analogue of the above expression, in turn, takes the form

$$
\begin{aligned}
& \mathbf{C}_{11}^{(5)}=\mathbf{C}_{11}^{(5)+}= \\
& -\frac{1}{2}\left(\mathbf{G}_{(1)} \widetilde{\mathbf{G}}_{(4)}^{+}+\widetilde{\mathbf{G}}_{(4)} \mathbf{G}_{(1)}^{+}+\mathbf{G}_{(2)} \widetilde{\mathbf{G}}_{(3)}^{+}+\widetilde{\mathbf{G}}_{(3)} \mathbf{G}_{(2)}^{+}\right) \\
& -\frac{1}{2}\left(\mathbf{C}_{11}^{(2)} \mathbf{C}_{11}^{(3)}+\mathbf{C}_{11}^{(3)} \mathbf{C}_{11}^{(2)}\right)
\end{aligned}
$$

and contains renormalization matrices of both second and third order.
Before finishing this appendix, let us dwell on solution of matrix equations conditioning our principal matrices $\widetilde{\mathbf{G}}_{(k)}$ and/or $\mathbf{G}_{(k)}$ and exemplified by Eqs. (A11) and (A14). We have to do here with equations of the general form

$$
\mathbf{A X}+\mathbf{X B}+\mathbf{C}=\mathbf{0},
$$

where $\mathbf{X}$ stands for the matrix being sought. The formal solution of Eq. (A26) is representable as an integral [64]. Application of the latter to express the matrix $\widetilde{\mathbf{G}}_{(k)}$ yields the result

$$
\widetilde{\mathbf{G}}_{(k)}=\int_{0}^{\infty} \exp \left(\mathbf{E}_{(+)} t\right) \widetilde{\mathbf{V}}_{(k)} \exp \left(\mathbf{E}_{(-)} t\right) \mathrm{d} t,
$$

which offers no local relations between elements of matrices $\widetilde{\mathbf{G}}_{(k)}$ and $\widetilde{\mathbf{V}}_{(k)}$. Given that $\mathbf{E}_{(+)}$and $\mathbf{E}_{(-)}$are diagonal matrices (i. e. the equality (38) is assumed), equations concerned may be solved algebraically. Separate elements $\widetilde{G}_{(k) i l}$ take then the form of fractions,

$$
\widetilde{G}_{(k) i l}=-\frac{\widetilde{V}_{(k) i l}}{\varepsilon_{(+) i}+\varepsilon_{(-) l}} .
$$

Substituting matrices $\mathbf{R}$ and $\mathbf{S G}_{(2)}-\mathbf{G}_{(2)} \mathbf{Q}$ for $\widetilde{\mathbf{V}}_{(1)}$ and $\widetilde{\mathbf{V}}_{(2)}$ respectively (see Eq. (A15)) yields the expressions for elements $G_{(1) i l}$ and $G_{(2) i l}$ exhibited in Eqs. (40) and (41).

\section{Appendix B}

One-electron density matrix as a projector to the subset of occupied localized MOs

Submatrices of the LMO representation matrix $\mathbf{C}$ are generally determined by matrix equations as discussed in Sec. 2 and in the Appendix A. This implies that elements of the matrix $\mathbf{C}$ are not expressible explicitly in contrast to the usual numerical forms of canonical MOs. Nevertheless, the indirect way of obtaining the CBO matrix $\mathbf{P}$ on the basis of projector to the entire subset of occupied LMOs does not lose its importance in the present case too. Moreover, application of this approach yields useful interdependences between matrices $\widetilde{\mathbf{G}}_{(k)}$ and $\mathbf{G}_{(k)}$ for $k=3,4, \ldots$ and thereby allows us to verify our principal relations shown in Eqs. (31)(37) directly.

Let us start with the general definition of the oneelectron DM of any molecular system as a projector to the relevant subset of occupied one-electron states 
(see e.g. [15]). Both canonical MOs and their noncanonical counterparts (including LMOs) of any type are able to play the role of one-electron orbitals here. Moreover, the projector itself is additionally multiplied by the occupation number 2 . Let the row-matrix of occupied LMOs (see Eq. (8)) be designated by the ketvector $\left|\Psi_{1}(\mathbf{r})\right\rangle$, where $\mathbf{r}$ represents the position of an electron in the real space. The above-specified definition of the DM $P\left(\mathbf{r} \mid \mathbf{r}^{\prime}\right)$ then takes the form

$$
P\left(\mathbf{r} \mid \mathbf{r}^{\prime}\right)=2\left|\Psi_{1}(\mathbf{r})\right\rangle\left\langle\Psi_{1}\left(\mathbf{r}^{\prime}\right)\right|,
$$

where $\left\langle\Psi_{1}\left(\mathbf{r}^{\prime}\right)\right|$ is the relevant bra-vector (columnmatrix). After substituting an expression for $\left|\Psi_{1}(\mathbf{r})\right\rangle$ like that of Eq. (8), we obtain

$$
P\left(\mathbf{r} \mid \mathbf{r}^{\prime}\right)=\sum_{I, J=1}^{2}\left|\Phi_{I}(\mathbf{r})\right\rangle \mathbf{P}_{I J}\left\langle\Phi_{J}\left(\mathbf{r}^{\prime}\right)\right|,
$$

where $\mathbf{P}_{I, J}(I=1,2 ; J=1,2)$ are multidimensional elements (blocks) of the representation of the DM in terms of two subsets of FOs $\left\{\Phi_{1}\right\}$ and $\left\{\Phi_{2}\right\}$ introduced in Sec. 2. The expression (B2) serves as a matrix generalization of the well-known bilinear form of the DM in terms of individual basis functions. For blocks $\mathbf{P}_{I J}$, we accordingly obtain

$$
\begin{aligned}
& \mathbf{P}_{11}=2 \mathbf{C}_{11} \mathbf{C}_{11}^{+}, \quad \mathbf{P}_{22}=2 \mathbf{C}_{21} \mathbf{C}_{21}^{+}, \\
& \mathbf{P}_{12}=2 \mathbf{C}_{11} \mathbf{C}_{21}^{+},
\end{aligned}
$$

where submatrices of the matrix $\mathbf{C}$ (see Eq. (7)) are contained.

Let us dwell first on submatrices $\mathbf{P}_{11}$ and $\mathbf{P}_{22}$ taking the diagonal positions in the CBO matrix $\mathbf{P}$. Diagonal elements of these submatrices $\left(P_{11, i i}\right.$ and $\left.P_{22, l l}\right)$ correspondingly coincide with populations (occupation numbers) of IOFOs $\left(X_{(+) i}\right)$ and of IVFOs $\left(X_{(-) l}\right)$. In this connection, let submatrices $\mathbf{P}_{11}$ and $\mathbf{P}_{22}$ be alternatively designated by $\mathbf{X}_{(+)}$and $\mathbf{X}_{(-)}$, respectively, and called the intrasubset population matrices. Substituting the power series for $\mathbf{C}_{11}$ shown in Eq. (10) into the first relation of Eq. (B3) followed by employment of the interdependences between $\mathbf{C}_{11}^{(k)}$ and $\mathbf{D}_{(+)}^{(k)}$ of Eq. (22) yields the result

$$
\mathbf{X}_{(+)}=2\left(\mathbf{I}-\mathbf{D}_{(+)}^{(2)}-\mathbf{D}_{(+)}^{(3)}-\mathbf{D}_{(+)}^{(4)}-\ldots\right)
$$

or

$$
\mathbf{X}_{(+)}=2\left(\mathbf{I}-\mathbf{D}_{(+)}\right) .
$$

The second relation of Eq. (B3) may be reformulated analogously. We obtain

$$
\mathbf{X}_{(-)}=2 \mathbf{D}_{(-)} .
$$

Thus, intrasubset population matrices prove to be proportional to respective intersubset delocalization matrices. The relations (B5) and (B6) evidently are matrix generalizations of Eqs. (31) and (32), respectively. Thus, passing to diagonal elements within the former serves to verify the latter directly.

Let us turn now to the last relation of Eq. (B3) determining the off-diagonal blocks of the matrix $\mathbf{P}$. Substituting the power series shown in Eq. (10) for both $\mathbf{C}_{11}$ and $\mathbf{C}_{21}^{+}$and collecting terms of the same order $(k)$ within the right-hand side of the relation concerned yields expressions for separate members $\left(\mathbf{P}_{12}^{(k)}\right)$ of the power series for the block $\mathbf{P}_{12}$. These are as follows:

$$
\begin{aligned}
& \mathbf{P}_{12}^{(0)}=\mathbf{0}, \quad \mathbf{P}_{12}^{(1)}=2 \mathbf{C}_{21}^{(1)+}, \quad \mathbf{P}_{12}^{(2)}=2 \mathbf{C}_{21}^{(2)+}, \\
& \mathbf{P}_{12}^{(3)}=2\left(\mathbf{C}_{21}^{(3)+}+\mathbf{C}_{11}^{(2)} \mathbf{C}_{21}^{(1)+}\right), \\
& \mathbf{P}_{12}^{(4)}=2\left(\mathbf{C}_{21}^{(4)+}+\mathbf{C}_{11}^{(2)} \mathbf{C}_{21}^{(2)+}+\mathbf{C}_{11}^{(3)} \mathbf{C}_{21}^{(1)+}\right),
\end{aligned}
$$

etc. Let us recall now that $\mathbf{C}_{21}^{(k)+}$ coincide with $-\widetilde{\mathbf{G}}_{(k)}$ for any $k$ as Eq. (12) indicates, whereas $\mathbf{P}_{12}^{(k)}$ equals to $-2 \mathbf{G}_{(k)}$ [10]. From the second and third relation of Eq. (B7) we then obtain that $\widetilde{\mathbf{G}}_{(1)}=\mathbf{G}_{(1)}$ and $\widetilde{\mathbf{G}}_{(2)}=\mathbf{G}_{(2)}$ as discussed already in Sec. 2. The last two relations of Eq. (B7), in turn, yield the following interdependences between matrices $\mathbf{G}_{(3)}$ and $\widetilde{\mathbf{G}}_{(3)}$, as well as between $\mathbf{G}_{(4)}$ and $\widetilde{\mathbf{G}}_{(4)}$ :

$$
\begin{gathered}
\mathbf{G}_{(3)}=\widetilde{\mathbf{G}}_{(3)}-\frac{1}{2} \mathbf{G}_{(1)} \mathbf{G}_{(1)}^{+} \mathbf{G}_{(1)}, \\
\mathbf{G}_{(4)}=\widetilde{\mathbf{G}}_{(4)}-\frac{1}{2}\left(\mathbf{G}_{(1)} \mathbf{G}_{(1)}^{+} \mathbf{G}_{(2)}\right. \\
\left.+\mathbf{G}_{(1)} \mathbf{G}_{(2)}^{+} \mathbf{G}_{(1)}+\mathbf{G}_{(2)} \mathbf{G}_{(1)}^{+} \mathbf{G}_{(1)}\right) .
\end{gathered}
$$

These relations allow a direct proof of our principal results of Eqs. (31)-(37). For instance, use of Eq. (B8) permits the expression of Eq. (18) for $2 d_{(+) i,(-) l}^{(4)}$ to be rewritten as follows:

$$
\begin{aligned}
2 d_{(+) i,(-) l}^{(4)}= & 4 G_{(1) i l} G_{(3) i l}+2\left|G_{(2) i l}\right|^{2} \\
& +2 G_{(1) i l}\left\{\mathbf{G}_{(1)} \mathbf{G}_{(1)}^{+} \mathbf{G}_{(1)}\right\}_{i l},
\end{aligned}
$$

where equalities $G_{(k) i l}^{*}=G_{(k) i l}$ and $\widetilde{G}_{(k) i l}^{*}=\widetilde{G}_{(k) i l}$ are additionally accepted. This formula coincides with that for $x_{(+) i,(-) l}^{(4)}$ derived previously [8, 9, 11] from solution of the commutation equation for the matrix $\mathbf{P}$. Thus, the first relation of Eq. (37) is verified for $k=4$. 
On the whole, interdependences like those of Eqs. (B8) and (B9) allow us to conclude the corrections $\mathbf{P}_{(k)}$ and $\mathbf{C}_{(k)}$ to be interrelated even for $k=3,4, \ldots$, although the relations concerned take somewhat more involved forms versus those established previously for $k=1$ and $k=2$ (Sec. 2).

\section{Appendix C}

\section{Derivation of expressions for total energies}

Let the Hamiltonian matrix of our system $(\mathbf{H})$ be block-diagonalized as shown in Eq. (5) and thereby to contain the eigenblocks $\mathbf{E}_{1}$ and $\mathbf{E}_{2}$ in its diagonal positions. The relevant total energy $(\mathcal{E})$ is then expressible as follows:

$$
\mathcal{E}=2 \operatorname{Tr} \mathbf{E}_{1},
$$

where the subscript 1 is assumed to refer to the subset of occupied LMOs $\left\{\Psi_{1}\right\}$. An alternative form of Eq. (C1) also results from Eq. (5), i. e.,

$$
\mathcal{E}=2 \operatorname{Tr}\left\{\mathbf{C}^{+} \mathbf{H C}\right\}_{11},
$$

where $\mathbf{C}$ is the $\mathrm{LMO}$ representation matrix. In our case, the matrix $\mathbf{H}$ is defined by Eqs. (1) and (2) and contains zero and first order members. This form of the initial Hamiltonian matrix may be substituted into Eq. (C2) along with the consequent power series for the matrix C (see Eq. (9)). Thereupon, terms of the same order $(k)$ may be collected to build up members of the power series for the submatrix $\left\{\mathbf{C}^{+} \mathbf{H C}\right\}_{11}$ and/or $\mathbf{E}_{1}$ and thereby for the resulting total energy $(\mathcal{E})$. The first two members of the latter series are

$$
\begin{aligned}
& \mathcal{E}_{(0)}=2 \operatorname{Tr}\left\{\mathbf{H}_{(0)}\right\}_{11}=2 \operatorname{Tr} \mathbf{E}_{(+)}, \\
& \mathcal{E}_{(1)}=2 \operatorname{Tr}\left\{\mathbf{H}_{(1)}+\mathbf{C}_{(1)}^{+} \mathbf{H}_{(0)}+\mathbf{H}_{(0)} \mathbf{C}_{(1)}\right\}_{11}=2 \operatorname{Tr} \mathbf{S},
\end{aligned}
$$

where $\mathbf{E}_{(+)}$and $\mathbf{S}$ are submatrices of our matrix $\mathbf{H}$ of Eq. (2). It is evident that the sum $\mathcal{E}_{(0)}+\mathcal{E}_{(1)}$ coincides with the total energy of isolated IOFOs.

Members of the same series of higher orders $(k=$ $2,3, \ldots)$ may be conveniently represented as sums of two components containing the zero order Hamiltonian matrix $\mathbf{H}_{(0)}$ and the relevant first order member $\mathbf{H}_{(1)}$, respectively. Let these components acquire additional superscripts $(\alpha)$ and $(\beta)$. For the $k$ th order corrections to the eigenblock $\mathbf{E}_{1}$ and to the total energy $\mathcal{E}$, we correspondingly obtain

$$
\mathbf{E}_{1(k)}=\mathbf{E}_{1(k)}^{(\alpha)}+\mathbf{E}_{1(k)}^{(\beta)}, \quad \mathcal{E}_{(k)}=\mathcal{E}_{(k)}^{(\alpha)}+\mathcal{E}_{(k)}^{(\beta)}
$$

and

$$
\mathcal{E}_{(k)}^{(\alpha)}=2 \operatorname{Tr} \mathbf{E}_{1(k)}^{(\alpha)}, \quad \mathcal{E}_{(k)}^{(\beta)}=2 \operatorname{Tr} \mathbf{E}_{1(k)}^{(\beta)} .
$$

Expressions for particular components $\mathbf{E}_{1(k)}^{(\alpha)}$ and $\mathbf{E}_{1(k)}^{(\beta)}$ are as follows:

$$
\mathbf{E}_{1(2)}^{(\alpha)}=\left\{\mathbf{C}_{(2)}^{+} \mathbf{H}_{(0)}+\mathbf{H}_{(0)} \mathbf{C}_{(2)}+\mathbf{C}_{(1)}^{+} \mathbf{H}_{(0)} \mathbf{C}_{(1)}\right\}_{11},
$$

$$
\begin{aligned}
\mathbf{E}_{1(2)}^{(\beta)}= & \left\{\mathbf{C}_{(1)}^{+} \mathbf{H}_{(1)}+\mathbf{H}_{(1)} \mathbf{C}_{(1)}\right\}_{11}, \\
\mathbf{E}_{1(3)}^{(\alpha)}= & \left\{\mathbf{C}_{(3)}^{+} \mathbf{H}_{(0)}+\mathbf{H}_{(0)} \mathbf{C}_{(3)}+\mathbf{C}_{(2)}^{+} \mathbf{H}_{(0)} \mathbf{C}_{(1)}\right. \\
& \left.+\mathbf{C}_{(1)}^{+} \mathbf{H}_{(0)} \mathbf{C}_{(2)}\right\}_{11}
\end{aligned}
$$

$$
\mathbf{E}_{1(3)}^{(\beta)}=\left\{\mathbf{C}_{(2)}^{+} \mathbf{H}_{(1)}+\mathbf{H}_{(1)} \mathbf{C}_{(2)}+\mathbf{C}_{(1)}^{+} \mathbf{H}_{(1)} \mathbf{C}_{(1)}\right\}_{11},
$$

etc. Let us invoke now Eqs. (2) and (7) and turn to separate blocks within the right-hand sides of Eqs. (C6)(C9). Thereupon, let us employ the expressions for separate submatrices of the corrections $\mathbf{C}_{(k)}$ derived in the Appendix A (see also Sec. 2). As a result, we obtain the following principal relations:

$$
(k-1) \mathcal{E}_{(k)}^{(\beta)}=-k \mathcal{E}_{(k)}^{(\alpha)}
$$

and

$$
\mathcal{E}_{(k)}^{(\alpha)}=-2 \operatorname{Tr}\left(\mathbf{D}_{(+)}^{(k)} \mathbf{E}_{(+)}+\mathbf{D}_{(-)}^{(k)} \mathbf{E}_{(-)}\right),
$$

where $\mathbf{D}_{(+)}^{(k)}$ and $\mathbf{D}_{(-)}^{(k)}$ are shown in Eq. (21). Use of Eqs. (C4), (C10), and (C11), in turn, yields an expression for the $k$ th order energy $\mathcal{E}_{(k)}$ in terms of intersubset delocalization matrices of the same order, namely,

$$
\mathcal{E}_{(k)}=\frac{2}{k-1} \operatorname{Tr}\left(\mathbf{D}_{(+)}^{(k)} \mathbf{E}_{(+)}+\mathbf{D}_{(-)}^{(k)} \mathbf{E}_{(-)}\right) .
$$

This expression indicates the energy alteration due to interorbital interaction to originate from the intersubset delocalization.

To demonstrate the derivation of Eqs. (C10)-(C12), let us start with the second order terms $(k=2)$. After substituting Eqs. (2) and (A13) into Eq. (C7), we obtain

$$
\mathbf{E}_{1(2)}^{(\beta)}=-\mathbf{R G}_{(1)}^{+}-\mathbf{G}_{(1)} \mathbf{R}^{+} .
$$

Similarly, Eqs. (2), (A13), (A16), and (C6) yield

$$
\mathbf{E}_{1(2)}^{(\alpha)}=-\frac{1}{2} \mathbf{G}_{(1)} \mathbf{G}_{(1)}^{+} \mathbf{E}_{(+)}
$$




$$
-\frac{1}{2} \mathbf{E}_{(+)} \mathbf{G}_{(1)} \mathbf{G}_{(1)}^{+}-\mathbf{G}_{(1)} \mathbf{E}_{(-)} \mathbf{G}_{(1)}^{+} .
$$

Let us invoke now the matrix equation (A11) and use it to replace the products $\mathbf{G}_{(1)}^{+} \mathbf{E}_{(+)}$and $\mathbf{E}_{(+)} \mathbf{G}_{(1)}$ of the first two terms of (C14) by $-\mathbf{E}_{(-)} \mathbf{G}_{(1)}^{+}-\mathbf{R}^{+}$and $-\mathbf{G}_{(1)} \mathbf{E}_{(-)}-\mathbf{R}$, respectively. Instead of Eq. (C14), we then obtain

$$
\mathbf{E}_{1(2)}^{(\alpha)}=\frac{1}{2}\left(\mathbf{R G}_{(1)}^{+}+\mathbf{G}_{(1)} \mathbf{R}^{+}\right) .
$$

Comparison of Eqs. (C13) and (C15) provides us with relations

$$
\mathbf{E}_{1(2)}^{(\beta)}=-2 \mathbf{E}_{1(2)}^{(\alpha)}, \quad \mathbf{E}_{1(2)}=-\mathbf{E}_{1(2)}^{(\alpha)},
$$

as well as with a compact expression for the second order correction to the eigenblock $\mathbf{E}_{1}$ originally derived in Ref. [65],

$$
\mathbf{E}_{1(2)}=-\frac{1}{2}\left(\mathbf{R G}_{(1)}^{+}+\mathbf{G}_{(1)} \mathbf{R}^{+}\right) .
$$

The relation (C10) for $k=2$ follows straightforwardly from Eq. (C16). Finally, Eqs. (21), (C4), and (C14) yield an expression for the second order energy $\mathcal{E}_{(2)}$ in terms of matrices $\mathbf{D}_{(+)}^{(2)}$ and $\mathbf{D}_{(-)}^{(2)}$, which coincides with Eq. (C12) for $k=2$ and may be found also in Ref. [66].

An analogous derivation may be performed for higher values of the order parameter $k$ too, although the relevant relations become somewhat more cumbersome. Let us consider the third order terms as an example. After invoking Eq. (A11) we obtain

$$
\begin{aligned}
\mathbf{E}_{1(3)}^{(\alpha)}=- & \frac{1}{2}\left(\mathbf{G}_{(1)} \mathbf{G}_{(2)}^{+} \mathbf{E}_{(+)}+\mathbf{E}_{(+)} \mathbf{G}_{(2)} \mathbf{G}_{(1)}^{+}\right. \\
& +\mathbf{G}_{(1)} \mathbf{E}_{(-)} \mathbf{G}_{(2)}^{+}+\mathbf{G}_{(2)} \mathbf{E}_{(-)} \mathbf{G}_{(1)}^{+} \\
& \left.-\mathbf{R G}_{(2)}^{+}-\mathbf{G}_{(2)} \mathbf{R}^{+}\right)
\end{aligned}
$$

instead of Eq. (C15). Meanwhile, the remaining component $\mathbf{E}_{1(3)}^{(\beta)}$ may be reformulated as

$$
\begin{aligned}
\mathbf{E}_{1(3)}^{(\beta)}= & \frac{1}{2}\left(\mathbf{G}_{(1)} \mathbf{G}_{(2)}^{+} \mathbf{E}_{(+)}+\mathbf{E}_{(+)} \mathbf{G}_{(2)} \mathbf{G}_{(1)}^{+}\right. \\
& \left.+\mathbf{G}_{(1)} \mathbf{E}_{(-)} \mathbf{G}_{(2)}^{+}+\mathbf{G}_{(2)} \mathbf{E}_{(-)} \mathbf{G}_{(1)}^{+}\right) \\
& -\left(\mathbf{R G}_{(2)}^{+}+\mathbf{G}_{(2)} \mathbf{R}^{+}\right)
\end{aligned}
$$

where Eqs. (A14) and (A15) are used to replace $\mathbf{S G}_{(1)}$ $\mathbf{G}_{(1)} \mathbf{Q}$ by $-\mathbf{E}_{(+)} \mathbf{G}_{(2)}-\mathbf{G}_{(2)} \mathbf{E}_{(-)}$. After summing up
Eqs. (C18) and (C19), the total third order correction to the eigenblock $\mathbf{E}_{1}$ takes the form

$$
\mathbf{E}_{1(3)}=-\frac{1}{2}\left(\mathbf{R G}_{(2)}^{+}+\mathbf{G}_{(2)} \mathbf{R}^{+}\right),
$$

as originally derived in Ref. [65]. Furthermore, employment of cyclic transpositions of matrices inside the $\operatorname{Tr}$ signs along with Eqs. (A11) and (C5) allows the components of the third order energy $\mathcal{E}_{(3)}$ to be represented in the following simple forms:

$$
\mathcal{E}_{(3)}^{(\alpha)}=4 \operatorname{Tr}\left(\mathbf{G}_{(2)} \mathbf{R}^{+}\right), \quad \mathcal{E}_{(3)}^{(\beta)}=-6 \operatorname{Tr}\left(\mathbf{G}_{(2)} \mathbf{R}^{+}\right) .
$$

The relation of Eq. (C10) for $k=3$ follows immediately after comparison of these expressions. Finally, invoking the definition of matrices $\mathbf{D}_{(+)}^{(3)}$ and $\mathbf{D}_{(-)}^{(3)}$ of Eq. (21) along with the initial formula for $\mathbf{E}_{1(3)}^{(\alpha)}$ resulting from Eq. (C8) allows the $\alpha$-component of the third order energy to be represented as shown in Eq. (C11). The total third order energy then takes the form of Eq. (C12) for $k=3$.

\section{References}

[1] Chemical Reactivity and Reaction Paths, ed. G. Klopman (John Wiley and Sons Inc., New York, London, Sydney, Toronto, 1974).

[2] J. March, Advanced Organic Chemistry, Reactions, Mechanisms and Structure, 3rd ed. (Wiley Interscience, New York, 1985).

[3] F.A. Carroll, Perspectives on Structure and Mechanism in Organic Chemistry, 6th ed. (Brooks/Cole, Pacific Grove, CA, 1996).

[4] H.G.O. Becker, Einfürung in die Elektronentheorie organisch-chemischen Reaktionen (Deutscher Verlag der Wissenschaften, Berlin, 1974).

[5] M. Edenborough, Organic Reaction Mechanisms. A Step by Step Approach, 2nd ed. (Taylor and Francis, London, 1999).

[6] A.S. Dneprovskii and T.I. Temnikova, Theoretical Fundamentals of Organic Chemistry (Khimiya, Leningrad, 1991) [in Russian].

[7] V. Gineityte, J. Mol. Struct. (Theochem) 588, 99 (2002).

[8] V. Gineityte, Int. J. Quant. Chem. 94, 302 (2003).

[9] V. Gineityte, Z. Naturforsch. A 64a, 132 (2009).

[10] V. Gineityte, J. Mol. Struct. (Theochem) 343, 183 (1995).

[11] V. Gineityte, J. Mol. Struct. (Theochem) 585, 15 (2002).

[12] C. Kaneko, Tetrahedron 28, 4915 (1972).

[13] S. Zumdahl, Chemical Principles, 5th ed. (Houghton Mifflin, Boston, 2005). 
[14] G.N. Lewis, J. Am. Chem. Soc. 38, 762 (1916).

[15] R. McWeeny, Methods in Molecular Ouantum Mechanics, 2nd ed. (Academic Press, London, 1989).

[16] L. Zülicke, Quantenchemie, Bd. 1, Grundlagen und algemeine Methoden (Deutscher Verlag der Wissenschaften, Berlin, 1973).

[17] G.L. Miesler and D.A. Tar, Inorganic Chemistry, 3rd ed. (Pearson Prentice-Hall, Indianapolis, 2003).

[18] B.B. Miburo, J. Chem. Educ. 75, 317 (1998).

[19] V. Gineityte, J. Mol. Struct. (Theochem) 810, 91 (2007).

[20] V.F. Traven, Electronic Structure and Properties of Organic Molecules (Khimiya, Moscow, 1989) [in Russian].

[21] S. Huzinaga, The MO Method (Mir, Moscow, 1983) [in Russian].

[22] F. Jensen, Introduction to Computational Chemistry (John Wiley and Sons, Chichester, 2007).

[23] S. Liu, J.M. Perez-Jorda, and W. Yang, J. Chem. Phys. 112, 1634 (2000).

[24] Z. Szekeres and P.R. Surjan, Chem. Phys. Lett. 369, 125 (2003).

[25] Localization and Delocalization in Quantum Chemistry, Atoms and Molecules in the Ground State, eds. O. Chalvet, R. Daudel, S. Diner, J.P. Malrieu, Vol. 1 (Reidel, Dordrecht, 1975).

[26] J.P. Daudey, Chem. Phys. Lett. 24, 574 (1974).

[27] I. Mayer, Chem. Phys. Lett. 89, 390 (1982).

[28] P.R. Surjan, I. Mayer, and M. Ketesz, J. Chem. Phys. 77, 2454 (1982).

[29] I. Mayer and P.R. Surjan, J. Chem. Phys. 80, 5649 (1984).

[30] C. Angeli and J.-P. Malrieu, J. Phys. Chem. A 112, 11481 (2008).

[31] J. Rubio, A. Povill, J.-P. Malrieu, and P. Reinhardt, J. Chem. Phys. 107, 10044 (1997).

[32] V.A. Kuprievich, Theor. Exp. Chem. 22, 245 (1986).

[33] H. Stoll, G. Wagenblast, and H. Preuß, Theor. Chim. Acta 57, 169 (1980).

[34] A. Fornili, M. Sironi, and M. Raimondi, J. Mol. Struct. (Theochem) 632, 157 (2003).

[35] C. Angeli, S. Evangelisti, R. Cimiraglia, and D. Maynau, J. Chem. Phys. 117, 10525 (2002).

[36] N. Guihery, J.-P. Malrieu, and S. Evangelisti, J. Chem. Phys. 119, 11088 (2003).

[37] A. Genoni and M. Sironi, Theor. Chem. Accounts 112, 254 (2004).

[38] S. Evangelisti, N. Guihery, T. Leininger, J.-P. Malrieu, D. Maynau, J.-V.P. Ruiz, N. Suaud, C. Angeli, R. Cimiraglia, and C.J. Calzado, J. Mol. Struct. (Theochem) 709, 1 (2004).
[39] J.-V.P. Ruiz, S. Evangelisti, and D. Maynau, Future Generat. Comput. Syst. 20, 821 (2004).

[40] J.J.P. Stewart, Int. J. Quant. Chem. 58, 133 (1996).

[41] V. Gineityte, J. Mol. Struct. (Theochem) 288, 111 (1993)

[42] V. Gineityte, J. Mol. Struct. (Theochem) 333, 297 (1995).

[43] S.F. Boys, Rev. Mod. Phys. 32, 296 (1960).

[44] C. Edmiston and K. Ruedenberg, Rev. Mod. Phys. 34, 457 (1963).

[45] C. Edmiston and K. Ruedenberg, J. Chem. Phys. 43, S97 (1965).

[46] J. Pipek and P.G. Mezey, J. Chem. Phys. 90, 4916 (1989).

[47] M.M. Mestetchkin, The Density Matrix Method in Quantum Chemistry (Naukova Dumka, Kiev, 1977) [in Russianl.

[48] V. Gineityte, Int. J. Quant. Chem. 68, 119 (1998).

[49] V. Gineityte, Lith. J. Phys. 44, 219 (2004).

[50] L.D. Landau and E.M. Lifshits, Quantum Mechanics (Non-relativistic Theory), 3rd ed. (ButterworthHeinemann, Oxford, 1977).

[51] V. Gineityte, Int. J. Quant. Chem. 72, 559 (1999).

[52] V. Gineityte, J. Mol. Struct. (Theochem) 546, 107 (2001).

[53] V. Gineityte, Int. J. Quant. Chem. 108, 1141 (2008).

[54] J.M. Tedder and A. Nechvatal, Pictorial Orbital Theory (Pitman, London 1985).

[55] M.J.S. Dewar and R.C. Dougherty, The PMO Theory of Organic Chemistry (Plenum Press, New York, 1975).

[56] V. Gineityte, J. Mol. Struct. (Theochem) 541, 1 (2001).

[57] C.K. Ingold, Structure and Mechanism in Organic Chemistry (Cornell University Press, Ithaca, 1953).

[58] V.I. Minkin, B.J. Simkin, and R.M. Mineev, Teoriya stroeniya molekul (Vysshaya Shkola, Moscow, 1979) [Theory of Molecular Structure, in Russian].

[59] A.R. Katritzky and C.D. Johnson, Angew. Chem. Int. Ed. 6, 608 (1967).

[60] V. Gineityte, J. Mol. Struct. (Theochem) 760, 229 (2006).

[61] V. Gineityte, J. Mol. Struct. (Theochem) 726, 205 (2005).

[62] V. Gineityte, J. Mol. Struct. (Theochem) 714, 157 (2005).

[63] V. Gineityte, J. Mol. Struct. (Theochem) 487, 231 (1999).

[64] P. Lancaster, Theory of Matrices (Academic Press, New York, 1969).

[65] V. Gineityte, Lith. J. Phys. 42, 397 (2002).

[66] V. Gineityte, Int. J. Quant. Chem. 77, 534 (2000). 


\title{
KRŪVIO PERSISKIRSTYMO IR DELOKALIZACIJOS TARPUSAVIO ATITIKIMAS IR JO TAIKYMAI ORGANINĖMS REAKCIJOMS TIRTI
}

\author{
V. Gineitytè
}

Vilniaus universiteto Teorinès fizikos ir astronomijos institutas, Vilnius, Lietuva

\section{Santrauka}

Krūvio persiskirstymas molekulèse ar molekulinèse sistemose dèl cheminès sąveikos dažnai yra siejamas su atskirų lokalizuotų elektronu poru daline delokalizacija. Klasikinėje chemijoje ši prielaida padeda interpretuojant organinių reakcijų tarpinių būsenų ar produktų santykinius stabilumus. Darbe siekiama pagrịsti tokią sąsają lyginant molekulinių sistemų vienelektronių tankio matricų ir jų nekanoninių (lokalizuotų) molekulinių orbitalių (MO) trikdžių teorijos eilutes tarpfragmentinių sąveikų atžvilgiu. Šiam tikslui apibrežti vadinamieji lokalizuotuju MO (LMO) delokalizacijos koeficientai ir nustatytas jų proporcingumas atitinkamų bazinių orbi- talių užpildymo skaičių pokyčiams dèl minètụjų sąveikų tikslumu iki penktos eilès narių imtinai. Gautieji rezultatai pritaikyti interpretuojant pagrindines organines reakcijas. Klasikinès šiu reakcijų ankstyvujų stadijų interpretacijos naudojantis vadinamosiomis lenktosiomis strèliukèmis dabar igauna kvantmechanini pagrindą. Be to, skirtingai nuo minètojo klasikinio interpretacijos būdo, darbe pasiūlytoji metodika igalina kiekybiškai palyginti alternatyvius tiriamosios reakcijos kelius pagal santykinius LMO delokalizacijos laipsnius. Tuo pagrindu parodyta, kad vyraujantys (leidžiami) reakcijų keliai pasižymi didesne LMO delokalizacija negu kiti (draudžiami) keliai. 